\title{
International Journal of Analysis and Applications
}

\section{An Extension of a Variational Inequality in the Simader Theorem to a Variable Exponent Sobolev Space and Applications: The Dirichlet Case}

\author{
Junichi Aramaki* \\ Division of Science, Tokyo Denki University, Hatoyama-machi, Saitama, 350-0394, Japan \\ *Corresponding author: aramaki@hctv.ne.jp
}

\begin{abstract}
In this paper, we shall extend a fundamental variational inequality which is developed by Simader in $W^{1, p}$ to a variable exponent Sobolev space $W^{1, p(\cdot)}$. The inequality is very useful for the existence theory to the Poisson equation with the Dirichlet boundary conditions in $L^{p(\cdot)}$-framework, where $L^{p(\cdot)}$ denotes a variable exponent Lebesgue space. Furthermore, we can also derive the existence of weak solutions to the Stokes problem in a variable exponent Lebesgue space.
\end{abstract}

\section{Introduction}

In Simader [25], the author derived a variational inequality of a bilinear form. More precisely, let $G$ is a bounded domain of $\mathbb{R}^{d}(d \geq 2)$ with a $C^{1}$-boundary $\partial G$ and $1<p<\infty$. The author proved that there exists a positive constant $C=C(p, G)>0$ such that

$$
\|\nabla u\|_{L^{p}(G)} \leq C \sup _{0 \neq v \in W_{0}^{1, p^{\prime}}(G)} \frac{\left|\langle\nabla u, \nabla v\rangle_{G}\right|}{\|\nabla v\|_{L^{p^{\prime}}(G)}} \text { for all } u \in W_{0}^{1, p}(G),
$$

where $\langle\nabla u, \nabla v\rangle_{G}=\int_{G} \nabla u \cdot \nabla v d x, \nabla$ denotes the gradient operator and $p^{\prime}$ is the conjugate exponent

of $p$, that is, $\frac{1}{p}+\frac{1}{p^{\prime}}=1$. He also considered the case where $G$ is an exterior domain and got a variational inequality like as in (1.1).

This inequality has many applications. For example, let $\boldsymbol{v} \in L^{p}(G)$, then it follows from (1.1) that the Dirichlet problem for the Poisson equation

$$
\begin{cases}\Delta u=\operatorname{div} v & \text { in } G \\ u=0 & \text { on } \partial G\end{cases}
$$

Received: Jan. 5, 2022.

2010 Mathematics Subject Classification. 35A15; 58E35; 35J25; 35D05; 35B30.

Key words and phrases. variational inequality; Dirichlet problem for the Poisson equation; the Helmholtz decomposition; the Stokes problem; variable exponent Sobolev spaces. 
has a unique solution in a generalized sense. The equation (1.2) plays an essential role for the existence of a solution to the Stokes problem (cf. Fujiwara and Morimoto [15] and Kozono and Yanagisawa [20]). It is also basic for the treatment of the Navier-Stokes equation, for example, see [15], Miyakawa [22].

In this paper, we attempt to derive an improvement of the above variational inequality (1.1) in the Sobolev opace $W_{0}^{1, p}(G)$ to a variable exponent Sobolev space $W_{0}^{1, p(\cdot)}(G)$ (Theorem 3.1). We restrict ourselves to the case where $G$ is a bounded domain. Though we follow the argument of [25], we have to proceed the analysis very carefully. The result brings about the existence theory of weak solutions to the Dirichlet problem for the Laplacian in the variable exponent Sobolev space, that is, for given functions $f \in W^{-1, p(\cdot)}(G)$ and $g \in \operatorname{Tr}\left(W^{1, p(\cdot)}(G)\right)$, where $W^{1, p(\cdot)}(G)$ is a variable exponent Sobolev space, $W^{-1, p(\cdot)}(G)$ is the dual space of $W_{0}^{1, p^{\prime}(\cdot)}(G)$ and $\operatorname{Tr}\left(W^{1, p(\cdot)}(G)\right)$ denotes the trace space,

$$
\begin{cases}-\Delta u=f & \text { in } G \\ u=g & \text { on } \partial G\end{cases}
$$

has a unique weak solution. According to our best knowledge, the result for the Dirichlet problem in a variable exponent Sobolev space is simplest. Furthermore, we show that the Stokes problem in a variable exponent Sobolev space has a unique weak (strong) solution by a new approach which is an application of Theorem 3.1.

The study of differential equations with $p(\cdot)$-growth conditions is a very interesting topic recently. Studying such problem stimulated its application in mathematical physics, in particular, in elastic mechanics (Zhikov [29]), in electrorheological fluids (Diening [10], Halsey [18], Mihăilescu and Rădulescu [21], Růžička [23]).

For the Neumann case of the variational inequality, we gave a result in the previous work Aramaki [5] (cf. Simader and Sohr [24] for the case $p(\cdot)=p=$ const.).

The paper is organized as follows. In section 2 , we give some preliminaries on variable exponent Lebesgue-Sobolev spaces. In section 3, we give main theorems (Theorem 3.1) which is an extension of variational inequality of type (1.1) to one in a variable exponent Sobolev space. Section 4 is a preparation of a proof of the main theorem. In section 5 , we give a proof of the main theorem. In section 6, we consider the Dirichlet problem of the Poisson equation. Finally, section 7 is devoted to the existence of a weak (strong) solution for the Stokes problem by a new approach.

\section{Preliminaries}

Throughout this paper, we only consider vector spaces of real valued functions over $\mathbb{R}$. For any space $B$, we denote $B^{d}$ by the boldface character $B$. Hereafter, we use this character to denote vectors and vector-valued functions, and we denote the standard inner product of vectors $\boldsymbol{a}=\left(a_{1}, \ldots, a_{d}\right)$ and $\boldsymbol{b}=\left(b_{1}, \ldots, b_{d}\right)$ in $\mathbb{R}^{d}$ by $\boldsymbol{a} \cdot \boldsymbol{b}=\sum_{i=1}^{d} a_{i} b_{i}$ and $|\boldsymbol{a}|=(\boldsymbol{a} \cdot \boldsymbol{a})^{1 / 2}$. Occasionally, we also use the same character for matrix values functions. Moreover, for the dual space $B^{\prime}$ of $B$ (resp. $B^{\prime}$ of $B$ ), we denote the duality bracket between $B^{\prime}$ and $B$ (resp. $\boldsymbol{B}^{\prime}$ and $\left.\boldsymbol{B}\right)$ by $\langle\cdot, \cdot\rangle_{B^{\prime}, B}\left(\operatorname{resp} .\langle\cdot, \cdot\rangle_{B^{\prime}, B}\right)$. 
In this section, we recall some well-known results on variable exponent Lebesgue-Sobolev spaces. See Diening et al. [11], Fan and Zhao [14], Fan and Zhang [12], Kováčik and Rácosnic [19] and references therein for more detail. Let $G$ be a (Lebesgue) measurable subset of $\mathbb{R}^{d}(d \geq 2)$ with the measure $|G|>0$. Then we define a set of variable exponents by $\mathcal{P}(G)=\{p ; G \rightarrow[1, \infty) ; p$ is measurable in $G\}$ and for $p \in \mathcal{P}(G)$, define

$$
p^{-}=\underset{x \in G}{\operatorname{essinf}} p(x) \text { and } p^{+}=\underset{x \in G}{\operatorname{esssup}} p(x) .
$$

For any real valued measurable function $u$ on $G$ and $p \in \mathcal{P}(G)$, a modular $\rho_{p(\cdot), G}$ is defined by

$$
\rho_{p(\cdot), G}(u)=\int_{G}|u(x)|^{p(x)} d x
$$

The variable exponent Lebesgue space is defined by

$$
L^{p(\cdot)}(G)=\left\{u ; u \text { is a measurable function on } G \text { satisfying } \rho_{p(\cdot), G}(u)<\infty\right\}
$$

equipped with the Luxemburg norm

$$
\|u\|_{L^{p(\cdot)}(G)}=\inf \left\{\lambda>0 ; \rho_{p(\cdot), G}\left(\frac{u}{\lambda}\right) \leq 1\right\} .
$$

Then $L^{p(\cdot)}(G)$ is a Banach space and it is separable if $p^{+}<\infty$ and reflexive if $1<p^{-} \leq p^{+}<\infty$. Define

$$
\mathcal{P}_{+}(G)=\left\{p \in \mathcal{P}(G) ; 1<p^{-} \leq p^{+}<\infty\right\}
$$

The following proposition is well known (see Fan et al. [13], Wei and Chen [26], [14], Zhao et al. [28], Yücedağ [27]).

Proposition 2.1. Let $G$ be a measurable set of $\mathbb{R}^{d}, p \in \mathcal{P}_{+}(G)$ and let $u, u_{n} \in L^{p(\cdot)}(G)(n=1,2, \ldots)$.

Then we have

(i) $\|u\|_{L^{p(\cdot)}(G)}<1(=1,>1) \Longleftrightarrow \rho_{p(\cdot), G}(u)<1(=1,>1)$.

(ii) $\|u\|_{L^{p(\cdot)}(G)}>1 \Longrightarrow\|u\|_{L^{p(\cdot)}(G)}^{p^{-}} \leq \rho_{p(\cdot), G}(u) \leq\|u\|_{L^{p(\cdot)}(G)}^{p^{+}}$.

(iii) $\|u\|_{L^{p(\cdot)}(G)}<1 \Longrightarrow\|u\|_{L^{p(\cdot)}(G)}^{p^{+}} \leq \rho_{p(\cdot), G}(u) \leq\|u\|_{L^{p(\cdot)}(G)}^{p^{-}}$.

(iv) $\lim _{n \rightarrow \infty}\left\|u_{n}-u\right\|_{L^{p(\cdot)}(G)}=0 \Longleftrightarrow \lim _{n \rightarrow \infty} \rho_{p(\cdot), G}\left(u_{n}-u\right)=0$.

(v) $\left\|u_{n}\right\|_{L^{p(\cdot)}(G)} \rightarrow \infty$ as $n \rightarrow \infty \Longleftrightarrow \rho_{p(\cdot), G}\left(u_{n}\right) \rightarrow \infty$ as $n \rightarrow \infty$.

The following proposition is a generalized Hölder inequality.

Proposition 2.2. Let $G$ be a measurable set of $\mathbb{R}^{d}$ and $p \in \mathcal{P}_{+}(G)$. For any $u \in L^{p(\cdot)}(G)$ and $v \in L^{p^{\prime}(\cdot)}(G)$, we have

$$
\int_{G}|u v| d x \leq\left(\frac{1}{p^{-}}+\frac{1}{\left(p^{\prime}\right)^{-}}\right)\|u\|_{L^{p(\cdot)}(G)}\|v\|_{L^{p^{\prime}(\cdot)}(G)} \leq 2\|u\|_{L^{p(\cdot)}(G)}\|v\|_{L^{p^{\prime}(\cdot)}(G)},
$$

where $p^{\prime}(\cdot)$ is the conjugate exponent of $p(\cdot)$, that is, $\frac{1}{p(x)}+\frac{1}{p^{\prime}(x)}=1$. 
When $G$ is a domain (open and connected subset) of $\mathbb{R}^{d}$ and $p \in \mathcal{P}_{+}(G)$, we can define a Sobolev space, for any integer $m \geq 0$,

$$
W^{m, p(\cdot)}(G)=\left\{u \in L^{p(\cdot)}(G) ; \partial^{\alpha} u \in L^{p(\cdot)}(G) \text { for }|\alpha| \leq m\right\},
$$

where $\alpha=\left(\alpha_{1}, \ldots, \alpha_{d}\right)$ is a multi-index, $|\alpha|=\sum_{i=1}^{d} \alpha_{i}, \partial^{\alpha}=\partial_{1}^{\alpha_{1}} \cdots \partial_{d}^{\alpha_{d}}$ and $\partial_{i}=\partial / \partial x_{i}$, endowed with the norm

$$
\|u\|_{W^{m, p(\cdot)}(G)}=\sum_{|\alpha| \leq m}\left\|\partial^{\alpha} u\right\|_{L^{p(\cdot)}(G)} .
$$

Of course, $W^{0, p(\cdot)}(G)=L^{p(\cdot)}(G)$. The local Sobolev space is defined by

$$
W_{\text {loc }}^{m, p(\cdot)}(G)=\left\{u ; \text { for all open subset } U \Subset G, u \in W^{m, p(\cdot)}(U)\right\} \text {, }
$$

where $U \Subset G$ means that the closure $\bar{U}$ of $U$ is compact and $\bar{U} \subset G$.

For $p \in \mathcal{P}_{+}(G)$, define

$$
p^{*}(x)= \begin{cases}\frac{d p(x)}{d-p(x)} & \text { if } p(x)<d \\ \infty & \text { if } p(x) \geq d\end{cases}
$$

and

$$
p^{\partial}(x)= \begin{cases}\frac{(d-1) p(x)}{d-p(x)} & \text { if } p(x)<d \\ \infty & \text { if } p(x) \geq d\end{cases}
$$

Proposition 2.3. Let $p \in \mathcal{P}_{+}(G)$ and $m \geq 0$ be an integer. Then we can see the following properties.

(i) The space $W^{m, p(\cdot)}(G)$ is a separable and reflexive Banach space.

(ii) Let $G$ be a bounded domain of $\mathbb{R}^{d}$. If $q(\cdot) \in \mathcal{P}_{+}(G)$ satisfies $q(x) \leq p(x)$ for all $x \in G$, then $W^{m, p(\cdot)}(G) \hookrightarrow W^{m, q(\cdot)}(G)$, where $\hookrightarrow$ means that the embedding is continuous.

(iii) Let $G$ be a bounded domain of $\mathbb{R}^{d}$. If $p, q \in \mathcal{P}_{+}(G) \cap C(\bar{G})$ satisfies that $q(x)<p^{*}(x)$ for all $x \in \bar{G}$, then the embedding $W^{1, p(\cdot)}(G) \hookrightarrow L^{q(\cdot)}(G)$ is compact.

Next we consider the trace. Let $G$ be a domain of $\mathbb{R}^{d}$ with a Lipschitz-continuous boundary $\partial G$ and $p \in \mathcal{P}_{+}(\bar{G})$. Since $W^{m, p(\cdot)}(G) \subset W_{\text {loc }}^{m, 1}(G)$, the trace $\gamma_{0}(u)=\left.u\right|_{\partial G}$ to $\partial G$ of any function $u$ in $W^{m, p(\cdot)}(G)$ is well defined as a function in $L_{\text {loc }}^{1}(\partial G)$. We define

$$
\operatorname{Tr}\left(W^{m, p(\cdot)}(G)\right)=\left\{f ; \gamma_{0}(u)=f \text { for some function } u \in W^{m, p(\cdot)}(G)\right\}
$$

equipped with the norm

$$
\|f\|_{\operatorname{Tr}\left(W^{m, p(\cdot)}(G)\right)}=\inf \left\{\|u\|_{W^{m, p(\cdot)}(G)} ; u \in W^{m, p(\cdot)}(G) \text { satisfying } \gamma_{0}(u)=f \text { on } \partial G\right\}
$$

for $f \in \operatorname{Tr}\left(W^{m, p(\cdot)}(G)\right)$. Then $\operatorname{Tr}\left(W^{m, p(\cdot)}(G)\right)$ is a Banach space. More precisely, see [11, Chapter 12]. We define a space

$$
\stackrel{\circ}{W}^{m, p(\cdot)}(G)=W^{m, p(\cdot)}(G) \cap W_{0}^{m, 1}(G) .
$$


For a general measurable subset $G$ of $\mathbb{R}^{d}$, we say that $p \in \mathcal{P}^{\log }(G)$ if $p \in \mathcal{P}_{+}(G)$ and $p$ has the globally log-Hölder continuity and globally log-Hölder decay condition in $G$, that is, $p: G \rightarrow \mathbb{R}$ satisfies that there exist a constant $C_{\log }(p)>0$ and $p_{\infty} \in \mathbb{R}$ such that the following inequalities hold:

$$
|p(x)-p(y)| \leq \frac{C_{\log }(p)}{\log (e+1 /|x-y|)} \text { for all } x, y \in G,
$$

and

$$
\left|p(x)-p_{\infty}\right| \leq \frac{C_{\log }(p)}{\log (e+|x|)} \text { for all } x \in G
$$

respectively.

Proposition 2.4. If $G$ is a domain of $\mathbb{R}^{d}$ and $p \in \mathcal{P}^{\log }(G)$, then $p$ has an extension $q \in \mathcal{P}^{\log }\left(\mathbb{R}^{d}\right)$ with $C_{\log }(q)=C_{\log }(p), q^{-}=p^{-}$and $q^{+}=p^{+}$. If $G$ is unbounded, then additionally $q_{\infty}=p_{\infty}$.

For the proof, see [11, Proposition 4.1.7].

We note that if $G$ is bounded, the global log-Hölder decay condition always holds. For a domain $G$, we write $\mathcal{P}_{+}^{\log }(G)=\mathcal{P}^{\log }(G) \cap \mathcal{P}_{+}(G)$. Let $G$ be a domain of $\mathbb{R}^{d}$ and $p \in \mathcal{P}_{+}^{\log }(G)$, define

$$
W_{0}^{m, p(\cdot)}(G)=\text { the closure of } C_{0}^{\infty}(G) \text { in } W^{m, p(\cdot)}(G) .
$$

From definition, we can easily see that $W_{0}^{m, p(\cdot)}(G) \subset \stackrel{\circ}{W}^{m, p(\cdot)}(G)$ and $\stackrel{\circ}{W}^{m, p(\cdot)}(G)$ is a closed subspace of $W^{m, p(\cdot)}(G)$. When $p(\cdot)=p$ is a constant, $W_{0}^{m, p}(G)=\stackrel{\circ}{W}^{m, p}(G)$, however, in general, $W_{0}^{m, p(\cdot)}(G) \subsetneq \stackrel{\circ}{W}^{m, p(\cdot)}(G)$.

Theorem 2.5. If $G$ is a bounded domain with a Lipschitz-continuous boundary $\partial G$ and $p \in \mathcal{P}_{+}^{\log }(\bar{G})$, then

(i) $C^{\infty}(\bar{G})$ is dense in $W^{m, p(\cdot)}(G)$.

(ii) $W_{0}^{m, p(\cdot)}(G)=\stackrel{\circ}{W}^{m, p(\cdot)}(G)$, In addition, when $m \geq 3$, assume that $G$ has a $C^{m, 1}$-boundary. Then we have

$$
W_{0}^{m, p(\cdot)}(G)=\left\{u \in W^{m, p(\cdot)}(\Omega) ; \gamma_{0}(u)=\cdots=\gamma_{m-1}(u)=0 \text { a.e. on } \partial G\right\},
$$

where $\gamma_{j}(u)=\frac{\partial^{j} u}{\partial \boldsymbol{n}^{j}}=\sum_{|\alpha|=j} \boldsymbol{n}^{\alpha} \partial^{\alpha} u, \boldsymbol{n}=\left(n_{1}, \ldots, n_{d}\right)$ is the outer unit normal vector to $\partial G$ and $n^{\alpha}=n_{1}^{\alpha_{1}} \cdots n_{d}^{\alpha_{d}}$.

For the proof, see [14, Theorem 2.6] and Galdi [16, Theorem 3.2].

Lemma 2.6. Let $G$ be a bounded domain of $\mathbb{R}^{d}$ with a Lipschitz-continuous boundary $\partial \Omega$ and let $p \in \mathcal{P}_{+}^{\log }(\bar{G})$. Assume that $q \in \mathcal{P}_{+}^{\log }(\partial \Omega)$ such that $q(x)<p^{\partial}(x)$ for all $x \in \partial G$. Then the trace operator $\operatorname{Tr}=\gamma_{0}: W^{1, p(\cdot)}(G) \rightarrow L^{q(\cdot)}(\partial G)$ is compact, In particular, $\operatorname{Tr}: W^{1, p(\cdot)}(G) \rightarrow L^{p(\cdot)}(\partial G)$ is compact.

For the proof, see Deng [9, Theorem 2.1].

Frequently we use the following Poincaré inequality later. 
Theorem 2.7. (i) If $G$ is a bounded domain of $\mathbb{R}^{d}$ and $p \in \mathcal{P}_{+}^{\log }(G)$, then there exists a constant $c$ depending only on $d$ and $C_{\log }(p)$ such that

$$
\|u\|_{L^{p(\cdot)}(G)} \leq c \operatorname{diam}(G)\|\nabla u\|_{L^{p(\cdot)}(G)} \text { for all } u \in W_{0}^{1, p(\cdot)}(G)
$$

where $\operatorname{diam}(G)$ denotes the diameter of $G$.

(ii) If $G$ is a bounded domain of $\mathbb{R}^{d}$ with a Lipschitz-continuous boundary $\partial G$ and $p \in \mathcal{P}_{+}^{\log }(G)$, then there exists a constant $c$ depending only on $d$ and $C_{\log }(p)$ such that

$$
\left\|u-\langle u\rangle_{G}\right\|_{L^{p(\cdot)}(G)} \leq c \operatorname{diam}(G)\|\nabla u\|_{L^{p(\cdot)}(G)} \text { for all } u \in W^{1, p(\cdot)}(G)
$$

where $\langle u\rangle_{G}=\frac{1}{|G|} \int_{G} u d x$

For the proof, see [11, Theorem 8.2.4].

Corollary 2.8. Let $G$ be a bounded domain of $\mathbb{R}^{d}$ with a Lipschitz-continuous boundary and let $p \in \mathcal{P}_{+}^{\log }(G)$. Furthermore, let $A \subset G$ such that $|A| \approx|G|$. Then there exists a constant $c$ depending only on $d$ and $C_{\log }(p)$ such that

$$
\left\|u-\langle u\rangle_{A}\right\|_{L^{p(\cdot)}(G)} \leq c \operatorname{diam}(G)\|\nabla u\|_{L^{p(\cdot)}(G)} \text { for all } u \in L_{\text {loc }}^{1}(G) \text { with } \nabla u \in L^{p(\cdot)}(G) \text {. }
$$

For the proof, see [11, Corollary 8.2.6].

We introduce a generalized Poincaré inequality which is found in Ciarlet and Dinca [8, Theorem 4.1].

Theorem 2.9. Let $G$ be a bounded domain of $\mathbb{R}^{d}$ with a Lipschitz-continuous boundary $\Gamma=\partial \Omega, G$ being locally on the same side of $\Gamma$. Moreover, let $\Gamma_{0}$ be a measurable subset of $\Gamma$ such that $\left|\Gamma_{0}\right|>0$, and let $p \in \mathcal{P}_{+}^{\log }(\bar{G})$. Define

$$
U=\left\{v \in W^{1, p(\cdot)}(G) ;\left.v\right|_{\Gamma_{0}}=0\right\} .
$$

Then there exists a constant $C=C(p, d, U)$ such that

$$
\|v\|_{L^{p(\cdot)}(G)} \leq C \operatorname{diam}(G)\|\nabla v\|_{L^{p(\cdot)}(G)} \text { for all } v \in U
$$

3. The weak Dirichlet problem for the Laplacian $\Delta$ in a variable exponent Sobolev space in a bounded domain

In this section, we state main theorems of this paper. Let $G$ be a bounded domain of $\mathbb{R}^{d}(d \geq 2)$ and $p \in \mathcal{P}_{+}^{\log }(G)$. Then taking the Poincare inequality (Theorem 2.7) into consideration, we may assume that the space $W_{0}^{1, p(\cdot)}(G)$ is equipped with the norm $\|\nabla v\|_{L^{p(\cdot)}(G)}$.

The first theorem is a variational inequality in $W_{0}^{1, p(\cdot)}(G)$. 
Theorem 3.1. Let $G$ be a bounded domain of $\mathbb{R}^{d}(d \geq 2)$ with a $C^{1}$-boundary and $p \in \mathcal{P}_{+}^{\log }(G)$. Then there exists a constant $C_{p}=C(p, G)>0$ such that

$$
\|\nabla u\|_{L^{p(\cdot)}(G)} \leq C_{p} \sup _{0 \neq v \in W_{0}^{1, p^{\prime}(\cdot)}(G)} \frac{\left|\langle\nabla u, \nabla v\rangle_{G}\right|}{\|\nabla v\|_{L^{p^{\prime}(\cdot)}(G)}} \text { for all } u \in W_{0}^{1, p(\cdot)}(G) .
$$

The second theorem is a functional representation in $W_{0}^{1, p(\cdot)}(G)$ which shows existence of weak solution to the Dirichlet problem of the Poisson equation in a bounded domain $G$.

Theorem 3.2. Let $G$ be a bounded domain of $\mathbb{R}^{d}(d \geq 2)$ with a $C^{1}$-boundary and $p \in \mathcal{P}_{+}^{\log }(G)$. For every $F^{\prime} \in\left(W_{0}^{1, p^{\prime}(\cdot)}(G)\right)^{\prime}=W^{-1, p(\cdot)}(G)$, there exists a unique $u \in W_{0}^{1, p(\cdot)}(G)$ such that

$$
F^{\prime}(v)=\langle\nabla u, \nabla v\rangle_{G} \text { for all } v \in W_{0}^{1, p^{\prime}(\cdot)}(G) \text {. }
$$

Furthermore, with $C_{p}>0$ in Theorem 3.1, the following inequality holds.

$$
C_{p}^{-1}\|\nabla u\|_{L^{p(\cdot)}(G)} \leq\left\|F^{\prime}\right\|_{\left(W_{0}^{1, p^{\prime}(\cdot)}(G)\right)^{\prime}} \leq 2\|\nabla u\|_{L^{p(\cdot)}(G)},
$$

where

$$
\left\|F^{\prime}\right\|_{\left(W_{0}^{1, p^{\prime}(\cdot)}(G)\right)^{\prime}}=\sup \left\{\left|F^{\prime}(v)\right| ; v \in W_{0}^{1, p^{\prime}(\cdot)}(G) \text { and }\|\nabla v\|_{L^{p^{\prime}(\cdot)}(G)} \leq 1\right\} .
$$

Before a proof of Theorem 3.1, we show that Theorem 3.1 implies Theorem 3.2. For this purpose, we use the following proposition (cf. Amrouche and Seloula [1, Theorem 4.2]).

Proposition 3.3. Let $X$ and $M$ be two reflexive Banach spaces, and a be a continuous bilinear form defined on $X \times M$. Assume that $A \in \mathcal{L}\left(X, M^{\prime}\right)$ and $A^{\prime} \in \mathcal{L}\left(M, X^{\prime}\right)$ are operators defined by

$$
\langle A u, v\rangle=\left\langle u, A^{\prime} v\right\rangle=a(u, v) \text { for } u \in X, v \in M
$$

and put $V=\operatorname{Ker} A$. Then the following statements are equivalent.

(i) There exists $\beta>0$ such that

$$
\inf _{0 \neq w \in M} \sup _{0 \neq v \in X} \frac{a(v, w)}{\|v\| x\|w\|_{M}} \geq \beta
$$

(ii) $A: X / V \rightarrow M^{\prime}$ is an isomorphism and $1 / \beta$ is the continuity constant of $A^{-1}$.

(iii) $A^{\prime}: M \rightarrow V^{\perp}:=\left\{f \in X^{\prime} ;\langle f, v\rangle=0\right.$ for all $\left.v \in V\right\}$ is also an isomorphism and $1 / \beta$ is the continuity constant of $\left(A^{\prime}\right)^{-1}$.

Let $X=\left(W_{0}^{1, p(\cdot)}(G),\|\nabla \cdot\|_{L^{p(\cdot)}(G)}\right)$ and $M=\left(W_{0}^{1, p^{\prime}(\cdot)}(G),\|\nabla \cdot\|_{L^{p^{\prime}(\cdot)}(G)}\right)$. Since $p, p^{\prime} \in \mathcal{P}_{+}^{\log }(G)$, $X$ and $M$ are reflexive Banach spaces (Proposition 2.3). Define

$$
a(u, v)=\langle\nabla u, \nabla v\rangle_{G}=\int_{G} \nabla u \cdot \nabla v d x \text { for } u \in X, v \in M .
$$

Then clearly $a$ is a bilinear form on $X \times M$, and it follows from the generalized Hölder inequality (Proposition 2.2) that $a$ is continuous. If $u \in \operatorname{Ker} A$, then $a(u, v)=\langle A u, v\rangle=0$ for all $v \in W_{0}^{1, p^{\prime}(\cdot)}(G)$. From Theorem 3.1, we have $\nabla u=0$ in $L^{p(\cdot)}(G)$. From the Poincaré inequality, we have $u=0$ in 
$L^{p(\cdot)}(G)$, so $\operatorname{Ker} A=\{0\}$. From Theorem 3.1, (i) in Proposition 3.3 holds with $\beta=1 / C_{p}$. Thus for any $F^{\prime} \in\left(W_{0}^{1, p^{\prime}(\cdot)}(G)\right)^{\prime}=M^{\prime}$, there exists uniquely $u \in W_{0}^{1, p(\cdot)}(G)$ such that $F^{\prime}=A u$, that is,

$$
F^{\prime}(v)=\langle A u, v\rangle=\langle\nabla u, \nabla v\rangle_{G} \text { for all } v \in W^{1, p^{\prime}(\cdot)}(G)
$$

and

$$
\|\nabla u\|_{L^{p(\cdot)}(G)} \leq \frac{1}{\beta}\left\|F^{\prime}\right\|_{\left(W_{0}^{1, p^{\prime}(\cdot)}(G)\right)^{\prime}}
$$

Therefore,

$$
C_{p}^{-1}\|\nabla u\|_{L^{p(\cdot)}(G)} \leq\left\|F^{\prime}\right\|_{\left(W_{0}^{1, p^{\prime} \cdot(\cdot)}(G)\right)^{\prime}} .
$$

Since by the generalized Hölder inequality (Proposition 2.2),

$$
\begin{aligned}
\left\|F^{\prime}\right\|_{\left(W_{0}^{1, p^{\prime}(\cdot)}(G)\right)^{\prime}} & =\sup \left\{\left|F^{\prime}(v)\right| ; v \in W_{0}^{1, p^{\prime}(\cdot)}(G),\|\nabla v\|_{L^{p^{\prime}(\cdot)}(G)} \leq 1\right\} \\
& =\sup \left\{\left|\langle\nabla u, \nabla v\rangle_{G}\right| ; v \in W_{0}^{1, p^{\prime}(\cdot)}(G),\|\nabla v\|_{L^{p^{\prime}(\cdot)}(G)} \leq 1\right\} \\
& \leq 2\|\nabla u\|_{L^{p(\cdot)}(G)}
\end{aligned}
$$

we can see that (3.3) holds.

\section{Preparation to a proof of Theorem 3.1}

We use the localization method for a proof of Theorem 3.1. For any open set $G \subset \mathbb{R}^{d}(d \geq 2)$ (not necessarily bounded), we say that $G$ satisfies (GA) if $G$ has a $C^{1}$-boundary and there exists a non-empty open set $K$ in $\mathbb{R}^{d}$ such that $G=\mathbb{R}^{d} \backslash \bar{K}$.

Definition 4.1. If $G$ satisfies $(G A)$ and $p \in \mathcal{P}_{+}^{\log }(G)$, define

$$
\begin{aligned}
\widehat{W}_{0}^{1, p(\cdot)}(G)= & \left\{v: G \rightarrow \mathbb{R} ; v \text { is measurable in } G, v \in L^{p(\cdot)}\left(G_{R}\right) \text { for each } R>0,\right. \\
& \nabla v \in L^{p(\cdot)}(G) \text {, there exists a sequence }\left\{v_{i}\right\}_{i=1}^{\infty} \subset C_{0}^{\infty}(G) \text { such that } \\
& \left.\left\|v-v_{i}\right\|_{L^{p(\cdot)}\left(G_{R}\right)} \rightarrow 0 \text { for each } R>0 \text { and }\left\|\nabla v-\nabla v_{i}\right\|_{L^{p(\cdot)}(G)} \rightarrow 0 \text { as } i \rightarrow \infty\right\},
\end{aligned}
$$

where $G_{R}=G \cap B_{R}, B_{R}=\left\{x \in \mathbb{R}^{d} ;|x|<R\right\}$.

Definition 4.2. If $G$ satisfies $(G A)$ and $p \in \mathcal{P}_{+}^{\log }(G)$, define

$$
\begin{aligned}
& \widehat{W}_{\bullet}^{1, p(\cdot)}(G)=\left\{v: G \rightarrow \mathbb{R} ; v \text { is measurable in } G, v \in L^{p(\cdot)}\left(G_{R}\right) \text { for each } R>0,\right. \\
& \left.\qquad \nabla v \in L^{p(\cdot)}(G) \text { and for any } \eta \in C_{0}^{\infty}\left(\mathbb{R}^{d}\right), \eta v \in W_{0}^{1, p(\cdot)}(G)\right\} .
\end{aligned}
$$

We note that if $G$ is bounded, then

$$
W_{0}^{1, p(\cdot)}(G)=\widehat{W}_{0}^{1, p(\cdot)}(G)=\widehat{W}_{\bullet}^{1, p(\cdot)}(G)
$$

We examine the properties of the spaces $\widehat{W}_{0}^{1, p(\cdot)}(G)$ and $\widehat{W}_{\bullet}^{1, p(\cdot)}(G)$. 
Lemma 4.3. Suppose $(G A)$. Let $p \in \mathcal{P}_{+}^{\log }(G)$ and let $v \in \widehat{W}_{\bullet}^{1, p(\cdot)}(G)$. Then for every $R>0$, there exists a sequence $\left\{v_{i}\right\} \subset C_{0}^{\infty}(G)$ possibly depending on $R>0$ such that

$$
\left\|v-v_{i}\right\|_{L^{p(\cdot)}\left(G_{R}\right)}+\left\|\nabla v-\nabla v_{i}\right\|_{L^{p(\cdot)}\left(G_{R}\right)} \rightarrow 0 \text { as } i \rightarrow \infty .
$$

Proof. For given $R>0$, choose $\eta \in C_{0}^{\infty}\left(B_{2 R}\right)$ such that $\eta=1$ on $B_{R}$. Since $\eta v \in W_{0}^{1, p(\cdot)}(G)$, there exists a sequence $\left\{v_{i}\right\} \subset C_{0}^{\infty}(G)$ such that $\left\|\eta v-v_{i}\right\|_{W^{1, p(\cdot)}(G)} \rightarrow 0$. Since $\eta=1$ on $B_{R}$, we have $\left\|v-v_{i}\right\|_{W^{1, p(\cdot)}\left(G_{R}\right)} \rightarrow 0$.

Theorem 4.4. Suppose $(G A)$ and let $p \in \mathcal{P}_{+}^{\log }(G)$. Then the following properties hold.

(i) $W_{0}^{1, p(\cdot)}(G) \subset \widehat{W}_{0}^{1, p(\cdot)}(G) \subset \widehat{W}_{\bullet}^{1, p(\cdot)}(G)$.

(ii) For $v \in \widehat{W}_{\bullet}^{1, p(\cdot)}(G),\|\nabla v\|_{L^{p(\cdot)}(G)}$ is a norm on $\widehat{W}_{\bullet}^{1, p(\cdot)}(G)$.

(iii) The space $\widehat{W}_{\bullet}^{1, p(\cdot)}(G)$ equipped with the norm $\|\nabla \cdot\|_{L^{p(\cdot)}}$ is a reflexive Banach space.

(iv) The space $\widehat{W}_{0}^{1, p(\cdot)}(G)$ is a closed subspace of $\widehat{W}_{\bullet}^{1, p(\cdot)}(G)$ and

$$
\widehat{W}_{0}^{1, p(\cdot)}(G)=\text { the closure of } C_{0}^{\infty}(G) \text { with respect to }\|\nabla \cdot\|_{L^{p(\cdot)}(G)} \text {-norm. }
$$

(v) The space $W_{0}^{1, p(\cdot)}(G)$ is dense in $\widehat{W}_{0}^{1, p(\cdot)}(G)$ with respect to $\|\nabla \cdot\|_{L^{p(\cdot)}(G)}$-norm.

(vi) If we define $E_{0}^{\infty}(G)=\left\{\nabla \phi ; \phi \in C_{0}^{\infty}(G)\right\}$ and $E_{0}^{p(\cdot)}(G)=\left\{\nabla v ; v \in \widehat{W}_{0}^{1, p(\cdot)}(G)\right\}$, then the closure of $E_{0}^{\infty}(G)$ in $L^{p(\cdot)}(G)$ is equal to $E_{0}^{p(\cdot)}(G)$.

Proof. (i) It is trivial that $W_{0}^{1, p(\cdot)}(G) \subset \widehat{W}_{0}^{1, p(\cdot)}(G)$. Let $v \in \widehat{W}_{0}^{1, p(\cdot)}(G)$ and let $\eta \in C_{0}^{\infty}\left(\mathbb{R}^{d}\right)$. Choose $R>0$ so that supp $\eta \subset B_{R}$. By definition of $\widehat{W}_{0}^{1, p(\cdot)}(G)$, there exists a sequence $\left\{v_{i}\right\} \subset C_{0}^{\infty}(G)$ such that $\left\|v-v_{i}\right\|_{L^{p(\cdot)}\left(G_{R}\right)} \rightarrow 0$ and $\left\|\nabla v-\nabla v_{i}\right\|_{L^{p(\cdot)}(G)} \rightarrow 0$ as $i \rightarrow \infty$. Then $\eta v_{i} \in C_{0}^{\infty}(G)$ and

$$
\begin{aligned}
\left\|\eta v-\eta v_{i}\right\|_{W^{1, p(\cdot)}(G)} \leq C_{1}\left(\|\eta\|_{L^{\infty}(G)}+\|\nabla \eta\|_{L^{\infty}(G)}\right)\left\|v-v_{i}\right\|_{L^{p(\cdot)}\left(G_{R}\right)} & \\
& +C_{2}\|\eta\|_{L^{\infty}(G)}\left\|\nabla v-\nabla v_{i}\right\|_{L^{p(\cdot)}(G)} \rightarrow 0 .
\end{aligned}
$$

Thus $\eta v \in W_{0}^{1, p(\cdot)}(G)$, so $v \in \widehat{W}_{\bullet}^{1, p(\cdot)}(G)$.

(ii) Clearly $\widehat{W}_{\bullet}^{1, p(\cdot)}(G)$ is a linear space. If $v \in \widehat{W}_{\bullet}^{1, p(\cdot)}(G)$ satisfies $\|\nabla v\|_{L^{p(\cdot)}(G)}=0$, then we show that $v=0$ in $G$. To do so, it suffices to show that $v=0$ in $G_{R}$ for every $R>0$. Choose $\eta \in C_{0}^{\infty}\left(\mathbb{R}^{d}\right)$ such that $\eta=1$ on $B_{R}$ and supp $\eta \subset B_{2 R}$. Since $\eta v \in W_{0}^{1, p(\cdot)}(G)$ by definition of $\widehat{W}_{\bullet}^{1, p(\cdot)}(G)$, there exists a sequence $\left\{v_{i}\right\} \subset C_{0}^{\infty}(G)$ such that $\left\|\eta v-v_{i}\right\|_{W^{1, p(\cdot)}(G)} \rightarrow 0$. Thus $\eta v \in W_{0}^{1, p^{-}}\left(G_{2 R}\right)$ and $\left\|\eta v-v_{i}\right\|_{W^{1, p^{-}}\left(G_{2 R}\right)} \rightarrow 0$. By [25, Lemma 1.2], we have

$$
\left\|v_{i}\right\|_{L^{p^{-}}\left(G_{2 R}\right)} \leq C R^{d / p^{-}+1-1 / p^{-}}\left\|\nabla v_{i}\right\|_{L^{p^{-}}\left(G_{2 R}\right)} .
$$

By the limit process, we have

$$
\|v\|_{L^{p^{-}}\left(G_{R}\right)} \leq C R^{d / p^{-}+1-1 / p^{-}}\|\nabla v\|_{L^{p^{-}}\left(G_{R}\right)} .
$$

Since $L^{p(\cdot)}\left(G_{R}\right) \hookrightarrow L^{p^{-}}\left(G_{R}\right)$ and $\nabla v=0$ in $L^{p^{-}}\left(G_{R}\right)$, we have $v=0$ in $G_{R}$. The other properties of norm clearly hold. 
(iii) We already showed that $\widehat{W}_{\bullet}^{1, p(\cdot)}(G)$ is a normed linear space. We derive the completeness of $\widehat{W}_{\bullet}^{1, p(\cdot)}(G)$ equipped with the norm $\|\nabla \cdot\|_{L^{p(\cdot)}(G)}$. Let $\left\{v_{i}\right\}_{i=1}^{\infty} \subset \widehat{W}_{\bullet}^{1, p(\cdot)}(G)$ be a Cauchy sequence, that is, $\left\|\nabla v_{i}-\nabla v_{j}\right\|_{L^{p(\cdot)}(G)} \rightarrow 0$ as $i, j \rightarrow \infty$. For $k \in \mathbb{N}$, put $G_{k}=G \cap B_{k}$. Then there exists $k_{0} \in \mathbb{N}$ such that for $k \geq k_{0}, G_{k}$ has a portion $\Gamma_{k}$ of $\partial G$ with $\left|\Gamma_{k}\right|>0$. Since $v_{i} \in W^{1, p(\cdot)}\left(G_{k}\right)$ and $\left.v_{i}\right|_{\Gamma_{k}}=0$, it follows from a generalized Poincaré inequality (Theorem 2.9) that

$$
\left\|v_{i}-v_{j}\right\|_{L^{p(\cdot)}\left(G_{k}\right)} \leq C(k)\left\|\nabla v_{i}-\nabla v_{j}\right\|_{L^{p(\cdot)}\left(G_{k}\right)} \leq C(k)\left\|\nabla v_{i}-\nabla v_{j}\right\|_{L^{p(\cdot)}(G)} .
$$

Thus $\left\{\left.v_{i}\right|_{G_{k}}\right\}$ is a Cauchy sequence in $L^{p(\cdot)}\left(G_{k}\right)$. Therefore, there exists $v^{(k)} \in L^{p(\cdot)}\left(G_{k}\right)$ such that $\left.v_{i}\right|_{G_{k}} \rightarrow v^{(k)}$ in $L^{p(\cdot)}\left(G_{k}\right)$. After choosing a subsequence, we may assume that $\left.v_{i}\right|_{G_{k}} \rightarrow v^{(k)}$ a.e. in $G_{k}$. Eventually after changing $v^{(k+1)}$ on a subset $N_{k} \subset G_{k}$ with measure zero, we may assume that $\left.v^{(k+1)}\right|_{G_{k}}=v^{(k)}$. Define a unique measurable function $v: G \rightarrow \mathbb{R}$ so that $v(x)=v^{(k)}(x)$ for $x \in G_{k}$. Hence for each $R>0\left\|v_{i}-v\right\|_{L^{p(\cdot)}\left(G_{R}\right)} \rightarrow 0$ as $i \rightarrow \infty$. Since $\left\{\nabla v_{i}\right\}$ is a Cauchy sequence in $L^{p(\cdot)}(G)$, there exists $\boldsymbol{f}=\left(f_{1}, \ldots, f_{d}\right) \in \boldsymbol{L}^{p(\cdot)}(G)$ such that $\nabla v_{i} \rightarrow \boldsymbol{f}$ in $\boldsymbol{L}^{p(\cdot)}(G)$. Let $\phi \in C_{0}^{\infty}(G)$. Then supp $\phi \subset G_{k}$ for some $k \in \mathbb{N}$. For $I=1, \ldots, d$,

$$
\left\langle v, \partial_{l} \phi\right\rangle_{G}=\lim _{i \rightarrow \infty}\left\langle v_{i}, \partial_{l} \phi\right\rangle_{G_{k}}=-\lim _{i \rightarrow \infty}\left\langle\partial_{l} v_{i}, \phi\right\rangle_{G_{k}}=-\left\langle f_{l}, \phi\right\rangle_{G}
$$

Hence $\partial_{l} v=f_{l} \in L^{p(\cdot)}(G)$, so $\nabla v=f \in L^{p(\cdot)}(G)$. For $\eta \in C_{0}^{\infty}\left(\mathbb{R}^{d}\right)$, choose $R>0$ such that supp $\eta \subset B_{R}$. Choose $\zeta \in C_{0}^{\infty}\left(B_{2 R}\right)$ so that $0 \leq \zeta \leq 1$ and $\zeta=1$ on $B_{R}$. Since $\zeta v_{i} \in W_{0}^{1, p(\cdot)}(G)$ by definition of $\widehat{W}_{\bullet}^{1, p(\cdot)}(G)$, there exists $\phi_{i} \in C_{0}^{\infty}(G)$ such that $\left\|\zeta v_{i}-\phi_{i}\right\|_{W^{1, p(\cdot)}(G)} \leq 2^{-i}$. Since $\eta v=\eta \zeta v$, we have

$$
\begin{aligned}
\left\|\eta v-\eta \phi_{i}\right\|_{L^{p(\cdot)}(G)} & =\left\|\eta \zeta v-\eta \phi_{i}\right\|_{L^{p(\cdot)}(G)} \\
& \leq\left\|\eta \zeta v-\eta \zeta v_{i}\right\|_{L^{p(\cdot)}(G)}+\left\|\eta \zeta v_{i}-\eta \phi_{i}\right\|_{L^{p(\cdot)}(G)} \\
& \leq\|\eta\|_{L^{\infty}\left(\mathbb{R}^{d}\right)}\left(\left\|\zeta v-\zeta v_{i}\right\|_{L^{p(\cdot)}(G)}+\left\|\zeta v_{i}-\phi_{i}\right\|_{L^{p(\cdot)}(G)}\right) \\
& \leq\|\eta\|_{L^{\infty}\left(\mathbb{R}^{d}\right)}\left(\left\|v-v_{i}\right\|_{L^{p(\cdot)}(G)}+2^{-i}\right) \rightarrow 0 \text { as } i \rightarrow \infty
\end{aligned}
$$

and

$$
\begin{aligned}
\left\|\nabla\left(\eta v-\eta \phi_{i}\right)\right\|_{L^{p(\cdot)}(G)}=\left\|\nabla\left(\eta \zeta v-\eta \phi_{i}\right)\right\|_{L^{p(\cdot)}(G)} \\
\leq\left\|\nabla\left(\eta \zeta v-\eta \zeta v_{i}\right)\right\|_{L^{p(\cdot)}(G)}+\left\|\nabla\left(\eta \zeta v_{i}-\eta \phi_{i}\right)\right\|_{L^{p(\cdot)}(G)} \\
\leq\|\eta \zeta\|_{L^{\infty}\left(\mathbb{R}^{d}\right)}\left(\left\|\nabla\left(v-v_{i}\right)\right\|_{L^{p(\cdot)}(G)}\right. \\
\quad+\|\nabla(\eta \zeta)\|_{L^{\infty}\left(\mathbb{R}^{d}\right)}\left\|v_{i}-\phi_{i}\right\|_{L^{p(\cdot)}\left(G_{R}\right)} \\
\quad+\left(\|\eta\|_{L^{\infty}\left(\mathbb{R}^{d}\right)}+\|\nabla \eta\|_{L^{\infty}\left(\mathbb{R}^{d}\right)}\right)\left\|\zeta v_{i}-\phi_{i}\right\|_{W^{1, p(\cdot)}(G)} \\
\rightarrow 0
\end{aligned}
$$

as $i \rightarrow \infty$. Since $\eta \phi \in C_{0}^{\infty}(G)$, we can see that $\eta v \in W_{0}^{1, p(\cdot)}(G)$, so $v \in \widehat{W}_{\bullet}^{1, p(\cdot)}(G)$. Hence $\widehat{W}_{\bullet}^{1, p(\cdot)}(G)$ is complete. 
We show the reflexivity of $\widehat{W}_{\bullet}^{1, p(\cdot)}(G)$. If we define

$$
E_{\bullet}^{p(\cdot)}(G)=\left\{\nabla v ; v \in \widehat{W}_{\bullet}^{1, p(\cdot)}(G)\right\}
$$

then the gradient operator $\nabla: \widehat{W}_{\bullet}^{1, p(\cdot)}(G) \rightarrow E_{\bullet}^{p(\cdot)}(G)$ is isometric isomorphism. Since $\widehat{W}_{\bullet}^{1, p(\cdot)}(G)$ is complete, $E_{\bullet}^{p(\cdot)}(G)$ is a closed subspace of a reflexive Banach space $L^{p(\cdot)}(G)$. Therefore, $E_{\bullet}^{p(\cdot)}(G)$ is reflexive, so $\widehat{W}_{\bullet}^{1, p(\cdot)}(G)$ is also reflexive.

(iv) Let $\left\{v_{i}\right\} \subset \widehat{W}_{0}^{1, p(\cdot)}(G)$ and $v \in \widehat{W}_{\bullet}^{1, p(\cdot)}(G)$ such that $\left\|\nabla v-\nabla v_{i}\right\|_{L^{p(\cdot)}(G)} \rightarrow 0$ as $i \rightarrow \infty$. By definition of $\widehat{W}_{0}^{1, p(\cdot)}(G)$, there exists $\phi_{i} \in C_{0}^{\infty}(G)$ such that $\left\|\nabla v_{i}-\nabla \phi_{i}\right\|_{L^{p(\cdot)}(G)} \leq 2^{-i}$. Hence

$$
\left\|\nabla v-\nabla \phi_{i}\right\|_{L^{p(\cdot)}(G)} \leq\left\|\nabla v-\nabla v_{i}\right\|_{L^{p(\cdot)}(G)}+\left\|\nabla v_{i}-\nabla \phi_{i}\right\|_{L^{p(\cdot)}(G)} \rightarrow 0 \text { as } i \rightarrow \infty \text {. }
$$

By the generalized Poincaré inequliaty (Theorem 2.9), for large $R>0$,

$$
\left\|v-\phi_{i}\right\|_{L^{p(\cdot)}\left(G_{R}\right)} \leq C(R)\left\|\nabla v-\nabla \phi_{i}\right\|_{L^{p(\cdot)}\left(G_{R}\right)} \leq C(R)\left\|\nabla v-\nabla \phi_{i}\right\|_{L^{p(\cdot)}(G)} \rightarrow 0 .
$$

From definition of $\widehat{W}_{0}^{1, p(\cdot)}(G)$, we can see that $v \in \widehat{W}_{0}^{1, p(\cdot)}(G)$, so $\widehat{W}_{0}^{1, p(\cdot)}(G)$ is a closed subspace of $\widehat{W}_{\bullet}^{1, p(\cdot)}(G)$.

Since $C_{0}^{\infty}(G) \subset \widehat{W}_{0}^{1, p(\cdot)}(G)$, the closure of $C_{0}^{\infty}(G)$ with respect to $\|\nabla \cdot\|_{L^{p(\cdot)}(G)}$ is contained in the closure of $\widehat{W}_{0}^{1, p(\cdot)}(G)$ with respect to $\|\nabla \cdot\|_{L^{p(\cdot)}(G)}$ which is equal to $\widehat{W}_{0}^{1, p(\cdot)}(G)$. Conversely, let $v \in \widehat{W}_{0}^{1, p(\cdot)}(G)$. Then there exists $\left\{\phi_{i}\right\} \subset C_{0}^{\infty}(G)$ such that $\left\|\nabla v-\nabla \phi_{i}\right\|_{L^{p(\cdot)}(G)} \rightarrow 0$ as $i \rightarrow \infty$. Thereby $v$ is contained in the closure of $C_{0}^{\infty}(G)$ with respect to $\|\nabla \cdot\|_{L^{p(\cdot)}(G)}$-norm.

(v) By definition of $\widehat{W}_{0}^{1, p(\cdot)}(G)$, the space $C_{0}^{\infty}(G)$ is dense in $\widehat{W}_{0}^{1, p(\cdot)}(G)$ with respect to $\|\nabla \cdot\|_{L^{p(\cdot)}(G)^{-}}$ norm. Since $C_{0}^{\infty}(G) \subset W_{0}^{1, p(\cdot)}(G) \subset \widehat{W}_{0}^{1, p(\cdot)}(G)$, we see that $W_{0}^{1, p(\cdot)}(G)$ is dense in $\widehat{W}_{0}^{1, p(\cdot)}(G)$ with respect to $\|\boldsymbol{\nabla} \cdot\|_{L^{p(\cdot)}(G)^{\text {-norm. }}}$

(vi) From (iv), it is clear that the closure of $E_{0}^{\infty}(G)$ in $L^{p(\cdot)}(G)$ is contained in $E_{0}^{p(\cdot)}(G)$. Let $v \in \widehat{W}_{0}^{1, p(\cdot)}(G)$. Then there exists a sequence $\left\{\phi_{i}\right\} \subset C_{0}^{\infty}(G)$ such that $\left\|\nabla v-\nabla \phi_{i}\right\|_{L^{p(\cdot)}(G)} \rightarrow 0$ as $i \rightarrow \infty$. Therefore, $\nabla v$ is contained in the closure of $E_{0}^{\infty}(G)$ in $L^{p(\cdot)}(G)$.

We can improve Lemma 4.3.

Lemma 4.5. Suppose $(G A)$. Let $p \in \mathcal{P}_{+}^{\log }(G)$ and let $v \in \widehat{W}_{\bullet}^{1, p(\cdot)}(G)$. Then there exists a sequence $\left\{v_{i}\right\} \subset C_{0}^{\infty}(G)$ such that for every $R>0$,

$$
\left\|v-v_{i}\right\|_{L^{p(\cdot)}\left(G_{R}\right)}+\left\|\nabla v-\nabla v_{i}\right\|_{L^{p(\cdot)}\left(G_{R}\right)} \rightarrow 0 \text { as } i \rightarrow \infty,
$$

that is, we can choose $\left\{v_{i}\right\} \subset C_{0}^{\infty}(G)$ independent of $R>0$.

Proof. Choose $\zeta \in C_{0}^{\infty}(G)$ such that $0 \leq \zeta \leq 1$, and $\zeta(x)=1$ for $|x| \leq 1$ and $\zeta=0$ for $|x| \geq 2$. Put $\zeta_{i}(x)=\zeta\left(i^{-1} x\right)$. By definition of $\widehat{W}_{\bullet}^{1, p(\cdot)}(G)$, we see that $\zeta_{i} v \in W_{0}^{1, p(\cdot)}(G)$. Hence there exists $\left\{v_{i}\right\} \subset C_{0}^{\infty}(G)$ such that $\left\|\zeta_{i} v-v_{i}\right\|_{W^{1, p(\cdot)}(G)} \leq i^{-1}$. For each $R>0$, let $i \geq R$. Then

$$
\left\|v-v_{i}\right\|_{L^{p(\cdot)}\left(G_{R}\right)}+\left\|\nabla v-\nabla v_{i}\right\|_{L^{p(\cdot)}\left(G_{R}\right)} \leq i^{-1} \rightarrow 0 \text { as } i \rightarrow \infty .
$$


Here we characterize of $\widehat{W}_{\bullet}^{1, p(\cdot)}(G)$.

Theorem 4.6. Suppose $(G A)$ and let $p \in \mathcal{P}_{+}^{\log }(G)$. Then we have $\widehat{W}_{\bullet}^{1, p(\cdot)}(G)=M_{p(\cdot)}$, where

$$
\begin{aligned}
& M_{p(\cdot)}=\left\{v: G \rightarrow \mathbb{R} ; v \text { is measurable, } v \in L^{p(\cdot)}\left(G_{R}\right) \text { for each } R>0,\right. \\
& \qquad \begin{array}{l}
\nabla v \in L^{p(\cdot)}(G) \text { and there exists }\left\{v_{i}\right\} \subset C_{0}^{\infty}(G) \text { such that } \\
\left.\quad\left\|v-v_{i}\right\|_{L^{p(\cdot)}\left(G_{R}\right)}+\left\|\nabla v-\nabla v_{i}\right\|_{L^{p(\cdot)}\left(G_{R}\right)} \rightarrow 0 \text { for each } R>0 \text { as } i \rightarrow \infty\right\} .
\end{array}
\end{aligned}
$$

Proof. By Lemma 4.5, $\widehat{W}_{\bullet}^{1, p(\cdot)}(G) \subset M_{p(\cdot)}$. Conversely, let $v \in M_{p(\cdot)}$, and let $\eta \in C_{0}^{\infty}\left(\mathbb{R}^{d}\right)$. Choose $R>0$ such that supp $\eta \subset B_{R}$. Then

$$
\left\|\eta v-\eta v_{i}\right\|_{L^{p(\cdot)}(G)} \leq\|\eta\|_{L^{\infty}\left(\mathbb{R}^{d}\right)}\left\|v-v_{i}\right\|_{L^{p(\cdot)}\left(G_{R}\right)} \rightarrow 0
$$

and

$$
\left\|\nabla\left(\eta v-\eta v_{i}\right)\right\|_{L^{p(\cdot)}(G)} \leq\|\eta\|_{L^{\infty}\left(\mathbb{R}^{d}\right)}\left\|\nabla v-\nabla v_{i}\right\|_{L^{p(\cdot)}\left(G_{R}\right)}+\|\nabla \eta\|_{L^{\infty}\left(\mathbb{R}^{d}\right)}\left\|v-v_{i}\right\|_{L^{p(\cdot)}\left(G_{R}\right)} \rightarrow 0 .
$$

] Since $\eta v_{i} \in C_{0}^{\infty}(G)$ and $\eta v_{i} \rightarrow \eta v$ in $W^{1, p(\cdot)}(G)$, we see that $\eta v \in W_{0}^{1, p(\cdot)}(G)$, so $v \in \widehat{W}_{\bullet}^{1, p(\cdot)}(G)$.

Definition 4.7. Let $G$ be a domain of $\mathbb{R}^{d}(d \geq 2)$ such that $\mathbb{R}^{d} \backslash \bar{G} \neq \emptyset$, and let $s \in \mathcal{P}_{+}^{\log }(G)$.

(a) We say that $G$ has the property $P_{a}(s)$ if there exists a constant $C_{s}=C(s, G)>0$ such that

$$
\|\nabla u\|_{L^{s(\cdot)}(G)} \leq C_{s} \sup _{0 \neq v \in \widehat{W}_{\bullet}^{1, s^{\prime}(\cdot)}(G)} \frac{\left|\langle\nabla u, \nabla v\rangle_{G}\right|}{\|\nabla v\|_{L^{s^{\prime}(\cdot)}(G)}} \text { for all } u \in \widehat{W}_{\bullet}^{1, s(\cdot)}(G) \text {. }
$$

(b) Let the bounded linear operator $\sigma_{s}: \widehat{W}_{\bullet}^{1, s(\cdot)}(G) \rightarrow\left(\widehat{W}_{\bullet}^{1, s^{\prime}(\cdot)}(G)\right)^{\prime}$ be defined by

$$
\sigma_{s}(u)(\phi)=\langle\nabla u, \nabla \phi\rangle_{G} \text { for } u \in \widehat{W}_{\bullet}^{1, s(\cdot)}(G) \text { and } \phi \in \widehat{W}_{\bullet}^{1, s^{\prime}(\cdot)}(G)
$$

We say that $G$ has the property $P_{b}(s)$ if $\sigma_{s}$ is a bijection and there exists a constant $\widetilde{C}_{s}=\widetilde{C}(s, G)>0$ such that

$$
\widetilde{C}_{s}\|\nabla u\|_{L^{s(\cdot)}(G)} \leq\left\|\sigma_{s}(u)\right\|_{\left(\widehat{W}_{\bullet}^{1, s^{\prime}(\cdot)}(G)\right)^{\prime}} \leq 2\|\nabla u\|_{L^{s(\cdot)}(G)} \text { for all } u \in \widehat{W}_{\bullet}^{1, s(\cdot)}(G) .
$$

Theorem 4.8. Let $G$ be a domain of $\mathbb{R}^{d}(d \geq 2)$ such that $\mathbb{R}^{d} \backslash \bar{G} \neq \emptyset$ and let $p \in \mathcal{P}_{+}^{\log }(G)$. Then $G$ has the property $P_{a}(s)$ for $s=p$ and $s=p^{\prime}$ if and only if $G$ has the property $P_{b}(s)$ for $s=p$ and $s=p^{\prime}$.

Proof. Assume that $G$ has the property $P_{a}(s)$ for $s=p$ and $s=p^{\prime}$. Let $u \in \widehat{W}_{\bullet}^{1, s(\cdot)}(G)$ and define

$$
S_{s}(u)=\sup \left\{\langle\nabla u, \nabla \phi\rangle_{G} ; \phi \in \widehat{W}_{\bullet}^{1, s^{\prime}(\cdot)}(G),\|\nabla \phi\|_{L^{s^{\prime}(\cdot)}(G)} \leq 1\right\} .
$$

By (4.1) and the Hölder inequality (Proposition 2.2),

$$
\begin{aligned}
C_{s}^{-1}\|\nabla u\|_{L^{s(\cdot)}(G)} \leq S_{s}(u) & =\left\|\sigma_{s}(u)\right\|_{\left(\widehat{W}_{\bullet}^{1, s^{\prime}(\cdot)}(G)\right)^{\prime}} \\
& \leq 2\|\nabla u\|_{L^{s(\cdot)}(G)} \text { for all } u \in \widehat{W}_{\bullet}^{1, s(\cdot)}(G) .
\end{aligned}
$$


Hence (4.3) holds with $\widetilde{C}_{s}=C_{s}^{-1}$. From this, we see that $\sigma_{s}\left(\widehat{W}_{\bullet}^{1, s^{\prime}(\cdot)}(G)\right)$ is a closed subspace of $\left(\widehat{W}_{\bullet}^{1, s^{\prime}(\cdot)}(G)\right)^{\prime}$. Suppose $\sigma_{s}\left(\widehat{W}_{\bullet}^{1, s(\cdot)}(G)\right) \subsetneq\left(\widehat{W}_{\bullet}^{1, s^{\prime}(\cdot)}(G)\right)^{\prime}$. By the Hahn-Banach theorem, there exists $F^{\prime \prime} \in\left(\widehat{W}_{\bullet}^{1, s^{\prime}(\cdot)}(G)\right)^{\prime \prime}$ such that $F^{\prime \prime} \neq 0$ and

$$
\left.F^{\prime \prime}\right|_{\sigma_{s}\left(\widehat{W}_{\bullet}^{1, s(\cdot)}(G)\right)}=0
$$

Since $\widehat{W}_{\bullet}^{1, s^{\prime}(\cdot)}(G)$ is reflexive, there exists uniquely $\phi \in \widehat{W}_{\bullet}^{1, s^{\prime}(\cdot)}(G)$ such that $F^{\prime \prime}\left(F^{\prime}\right)=F^{\prime}(\phi)$ for all $F^{\prime} \in\left(\widehat{W}_{\bullet}^{1, s^{\prime}(\cdot)}(G)\right)^{\prime}$ and $\left\|F^{\prime \prime}\right\|_{\left(\widehat{W}_{\bullet}^{1, s^{\prime}(\cdot)}(G)\right)^{\prime \prime}}=\|\nabla \phi\|_{L^{s^{\prime}(\cdot)}(G)}>0$. On the other hand, for all $u \in$ $\widehat{W}_{\bullet}^{1, s(\cdot)}(G)$,

$$
0=F^{\prime \prime}\left(\sigma_{s}(u)\right)=\sigma_{s}(u)(\phi)=\langle\nabla u, \nabla \phi\rangle_{G} .
$$

By the property $P_{a}\left(s^{\prime}\right)$, we have $\|\nabla \phi\|_{L^{s^{\prime}(\cdot)}(G)} \leq C_{s^{\prime}} S_{s^{\prime}}(\phi)=0$. This is a contradiction.

Conversely, assume that $G$ has the property $P_{b}(s)$ for $s=p$ and $s=p^{\prime}$. Let $u \in \widehat{W}_{\bullet}^{1, s(\cdot)}(G)$. Since $\sigma_{s^{\prime}}$ is a bijection, for $F^{\prime} \in\left(\widehat{W}_{\bullet}^{1, s(\cdot)}(G)\right)^{\prime}$, there exists uniquely $\phi \in \widehat{W}_{\bullet}^{1, s^{\prime}(\cdot)}(G)$ such that $F^{\prime}=\sigma_{s^{\prime}}(\phi)$. Hence

$$
\begin{aligned}
\|\nabla u\|_{L^{s(\cdot)}(G)} & =\sup \left\{\frac{\left|F^{\prime}(u)\right|}{\left\|F^{\prime}\right\|_{\left(\widehat{W}_{\bullet}^{1, s(\cdot)}(G)\right)^{\prime}}} ; 0 \neq F^{\prime} \in\left(\widehat{W}_{\bullet}^{1, s(\cdot)}(G)\right)^{\prime}\right\} \\
& \leq \sup \left\{\frac{\left|\langle\nabla u, \nabla \phi\rangle_{G}\right|}{\widetilde{C}_{s^{\prime}}\|\nabla \phi\|_{L^{s^{\prime}(\cdot)}(G)}} ; 0 \neq \phi \in \widehat{W}_{\bullet}^{1, s^{\prime}(\cdot)}(G)\right\} .
\end{aligned}
$$

Thus (4.1) holds with $C_{s}=\widetilde{C}_{s^{\prime}}^{-1}$.

Now we consider the case $G=\mathbb{R}^{d}$.

Lemma 4.9. If we define $M:=\left\{\Delta v ; v \in \mathcal{D}\left(\mathbb{R}^{d}\right):=C_{0}^{\infty}\left(\mathbb{R}^{d}\right)\right\}$, then $M$ is dense in $L^{p(\cdot)}\left(\mathbb{R}^{d}\right)$.

Proof. Suppose that $\bar{M} \varsubsetneqq L^{p(\cdot)}\left(\mathbb{R}^{d}\right)$, where $\bar{M}$ is the closure of $M$ in $L^{p(\cdot)}\left(\mathbb{R}^{d}\right)$. By the HahnBanach theorem, there exists $F^{\prime} \in\left(L^{p(\cdot)}\left(\mathbb{R}^{d}\right)\right)^{\prime}$ with $\left\|F^{\prime}\right\|_{\left(L^{p(\cdot)}\left(\mathbb{R}^{d}\right)\right)^{\prime}}>0$ and $\left.F^{\prime}\right|_{\bar{M}}=0$. Since we can

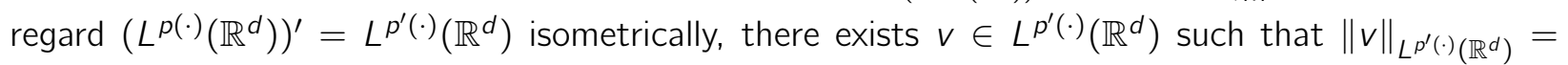
$\left\|F^{\prime}\right\|_{\left(L^{p(\cdot)}\left(\mathbb{R}^{d}\right)\right)^{\prime}}>0$ and $F^{\prime}(w)=\langle v, w\rangle_{\mathbb{R}^{d}}:=\int_{\mathbb{R}^{d}} v(x) w(x) d x$ for all $w \in L^{p(\cdot)}\left(\mathbb{R}^{d}\right)$. Since $\left.F^{\prime}\right|_{\bar{M}}=0$, we can see that $\langle v, \Delta \phi\rangle_{\mathbb{R}^{d}}=0$ for all $\phi \in \mathcal{D}\left(\mathbb{R}^{d}\right)$, so $\Delta v=0$ in $\mathcal{D}^{\prime}\left(\mathbb{R}^{d}\right)$. By the hypoellipticity of the Laplacian, we can regard that $v \in C^{\infty}\left(\mathbb{R}^{d}\right)$ (eventually after change of a set of measure zero), so $v$ is harmonic in $\mathbb{R}^{d}$. For any $x \in \mathbb{R}^{d}$ fixed, it follows from the second mean value theorem for harmonic functions that

$$
v(x)=\frac{1}{\left|B_{R}(x)\right|} \int_{B_{R}(x)} v(y) d y,
$$

where $B_{R}(x)=\left\{y \in \mathbb{R}^{d}:|y-x|<R\right\}$ and $\left|B_{R}(x)\right|$ denotes the volume of $B_{R}(x)$. By the generalized Hölder inequality (Proposition 2.2),

$$
|v(x)| \leq \frac{2}{\left|B_{R}(x)\right|}\|v\|_{L^{p^{\prime}(\cdot)}\left(B_{R}(x)\right)}\|1\|_{L^{p(\cdot)}\left(B_{R}(x)\right)} .
$$


Since $\|1\|_{L^{p(\cdot)}\left(B_{R}(x)\right)} \leq \rho_{p(\cdot), B_{R}(x)}(1)^{1 / p^{-}}=\left|B_{R}(x)\right|^{1 / p^{-}}$for large $R>0$ and $p^{-}>1$, we have

$$
|v(x)| \leq 2\left|B_{R}(x)\right|^{-1+1 / p^{-}}\|v\|_{L^{p^{\prime}(\cdot)\left(\mathbb{R}^{d}\right)}} \rightarrow 0 \text { as } R \rightarrow \infty .
$$

Therefore, we have $v(x)=0$. Since $x \in \mathbb{R}^{d}$ is arbitrary, $v \equiv 0$ in $\mathbb{R}^{d}$. This is a contradiction.

Define $\nabla^{2} v=\left(\partial_{i} \partial_{j} v\right)_{i, j=1, \ldots, d}$ for $v \in \mathcal{D}\left(\mathbb{R}^{d}\right)$. Then there exists a constant $C=C(p, d)>0$ such that

$$
C\left\|\nabla^{2} v\right\|_{L^{p(\cdot)}\left(\mathbb{R}^{d}\right)} \leq\|\Delta v\|_{L^{p(\cdot)}\left(\mathbb{R}^{d}\right)} \text { for all } v \in \mathcal{D}\left(\mathbb{R}^{d}\right) .
$$

For the proof, see [11, Corollary 14.1.7] (cf. when $p(\cdot)=p$ (constant), see Gilbarg and Trudinger [17, Corollary 9.10]).

For $p \in \mathcal{P}_{+}^{\log }\left(\mathbb{R}^{d}\right)$, we have $E^{p(\cdot)}\left(\mathbb{R}^{d}\right)=\left\{\nabla u ; u \in L_{\text {loc }}^{p(\cdot)}\left(\mathbb{R}^{d}\right), \nabla u \in L^{p(\cdot)}\left(\mathbb{R}^{d}\right)\right\}$ by definition.

Lemma 4.10. Let $p, q \in \mathcal{P}_{+}^{\log }\left(\mathbb{R}^{d}\right)$. If $u \in L_{\text {loc }}^{q(\cdot)}\left(\mathbb{R}^{d}\right)$ with $\nabla u \in L^{q(\cdot)}\left(\mathbb{R}^{d}\right)$ satisfies

$$
\sup _{0 \neq v \in \mathcal{D}\left(\mathbb{R}^{d}\right)} \frac{\left|\langle\nabla u, \nabla v\rangle_{\mathbb{R}^{d}}\right|}{\|\nabla v\|_{L^{p^{\prime}(\cdot)}\left(\mathbb{R}^{d}\right)}}<\infty
$$

then $u \in L_{\text {loc }}^{p(\cdot)}\left(\mathbb{R}^{d}\right)$ with $\nabla u \in L^{p(\cdot)}\left(\mathbb{R}^{d}\right)$. Furthermore, there exists a constant $C_{1}=C_{1}(p, d)>0$ such that

$$
\|\nabla u\|_{L^{p(\cdot)}\left(\mathbb{R}^{d}\right)} \leq C_{1} \sup _{0 \neq v \in \mathcal{D}\left(\mathbb{R}^{d}\right)} \frac{\left|\langle\nabla u, \nabla v\rangle_{\mathbb{R}^{d}}\right|}{\|\nabla v\|_{L^{p^{\prime}(\cdot)}\left(\mathbb{R}^{d}\right)}}
$$

for all $u \in L_{\text {loc }}^{p(\cdot)}\left(\mathbb{R}^{d}\right)$ with $\nabla u \in L^{p(\cdot)}\left(\mathbb{R}^{d}\right)$.

In particular, if $u \in L_{\text {loc }}^{p(\cdot)}\left(\mathbb{R}^{d}\right)$ with $\nabla u \in L^{p(\cdot)}\left(\mathbb{R}^{d}\right)$, then

$$
\|\nabla u\|_{L^{p(\cdot)}\left(\mathbb{R}^{d}\right)} \leq C_{1} \sup _{0 \neq v \in \mathcal{D}\left(\mathbb{R}^{d}\right)} \frac{\left|\langle\nabla u, \nabla v\rangle_{\mathbb{R}^{d}}\right|}{\|\nabla v\|_{L^{p^{\prime}(\cdot)}\left(\mathbb{R}^{d}\right)}}
$$

Proof. Let $u \in L_{\text {loc }}^{q(\cdot)}\left(\mathbb{R}^{d}\right)$ with $\nabla u \in L^{q(\cdot)}\left(\mathbb{R}^{d}\right)$. For every $i=1, \ldots, d$, using (4.4),

$$
\begin{aligned}
& \infty>\sup _{0 \neq v \in \mathcal{D}\left(\mathbb{R}^{d}\right)} \frac{\left|\langle\nabla u, \nabla v\rangle_{\mathbb{R}^{d}}\right|}{\|\nabla v\|_{L^{p^{\prime}(\cdot)}\left(\mathbb{R}^{d}\right)}} \\
& \geq \sup _{0 \neq w \in \mathcal{D}\left(\mathbb{R}^{d}\right)} \frac{\left|\left\langle\nabla u, \nabla\left(\partial_{i} w\right)\right\rangle_{\mathbb{R}^{d}}\right|}{\left\|\nabla \partial_{i} w\right\|_{L^{p^{\prime}(\cdot)}\left(\mathbb{R}^{d}\right)}} \\
& \geq \sup _{0 \neq w \in \mathcal{D}\left(\mathbb{R}^{d}\right)} \frac{\left|\left\langle\partial_{i} u, \Delta w\right\rangle_{\mathbb{R}^{d}}\right|}{\left\|\nabla^{2} w\right\|_{L^{p^{\prime}(\cdot)}\left(\mathbb{R}^{d}\right)}} \\
& \geq C \sup _{0 \neq w \in \mathcal{D}\left(\mathbb{R}^{d}\right)} \frac{\left|\left\langle\partial_{i} u, \Delta w\right\rangle_{\mathbb{R}^{d}}\right|}{\|\Delta w\|_{L^{p^{\prime}(\cdot)}\left(\mathbb{R}^{d}\right)}}
\end{aligned}
$$

where $C$ is the constant in (4.4). Define a bounded linear functional $L^{*}$ by

$$
L^{*}(\Delta w)=\left\langle\partial_{i} u, \Delta w\right\rangle_{\mathbb{R}^{d}} \text { on the dense subspace } M \text { of } L^{p^{\prime}(\cdot)}\left(\mathbb{R}^{d}\right)
$$


(cf. Lemma 4.9). Then the functional $L^{*}$ has a unique and norm-preserving extension as a continuous linear functional on $L^{p^{\prime}(\cdot)}\left(\mathbb{R}^{d}\right)$. Thus there exists $g \in L^{p(\cdot)}\left(\mathbb{R}^{d}\right)$ such that $\left\langle\partial_{i} u, v\right\rangle_{\mathbb{R}^{d}}=\langle g, v\rangle_{\mathbb{R}^{d}}$ for all $v \in M$, that is,

$$
\left\langle\partial_{i} u-g, \Delta w\right\rangle_{\mathbb{R}^{d}}=0 \text { for all } w \in \mathcal{D}\left(\mathbb{R}^{d}\right) .
$$

If we define $W=\partial_{i} u-g$, then $\Delta W=0$ in $\mathcal{D}^{\prime}\left(\mathbb{R}^{d}\right)$, so $W$ is harmonic in $\mathbb{R}^{d}$. By the same argument as in the proof of Lemma 4.9, we can regard $W(x) \equiv 0$, so $\partial_{i} u=g \in L^{p(\cdot)}\left(\mathbb{R}^{d}\right)$. Hence we have $\nabla u \in L^{p(\cdot)}\left(\mathbb{R}^{d}\right)$. Since $\nabla u \in L^{p(\cdot)}\left(\mathbb{R}^{d}\right)$ and $u \in L_{\text {loc }}^{q(\cdot)}\left(\mathbb{R}^{d}\right) \subset L_{\text {loc }}^{1}\left(\mathbb{R}^{d}\right)$, for any ball $B$, it follows from Theorem 2.7 (ii) that

$$
\left\|u-\langle u\rangle_{B}\right\|_{L^{p(\cdot)}(B)} \leq C(p, d, B)\|\nabla u\|_{L^{p(\cdot)}(B)} .
$$

This implies that $u \in L_{\text {loc }}^{p(\cdot)}\left(\mathbb{R}^{d}\right)$, that is, $\nabla u \in E^{p(\cdot)}\left(\mathbb{R}^{d}\right)$. By continuity, we have

$$
\sup _{0 \neq w \in \mathcal{D}\left(\mathbb{R}^{d}\right)} \frac{\left|\left\langle\partial_{i} u, \Delta w\right\rangle_{\mathbb{R}^{d}}\right|}{\|\Delta w\|_{L^{p^{\prime}(\cdot)}\left(r r^{d}\right)}}=\sup _{0 \neq f \in L^{p^{\prime}(\cdot)}\left(\mathbb{R}^{d}\right)} \frac{\left|\left\langle\partial_{i} u, f\right\rangle_{\mathbb{R}^{d}}\right|}{\|f\|_{L^{p^{\prime}(\cdot)}\left(r r^{d}\right)}}=\left\|\partial_{i} u\right\|_{L^{p(\cdot)}\left(\mathbb{R}^{d}\right)} .
$$

From (4.8),

$$
\left\|\partial_{i} u\right\|_{L^{p(\cdot)\left(\mathbb{R}^{d}\right)}} \leq C_{p^{\prime}}^{-1} \sup _{0 \neq \phi \in \mathcal{D}\left(\mathbb{R}^{d}\right)} \frac{\left|\langle\nabla u, \nabla \phi\rangle_{\mathbb{R}^{d}}\right|}{\|\nabla \phi\|_{L^{p^{\prime}(\cdot)}\left(\mathbb{R}^{d}\right)}}
$$

Therefore, we get (4.6).

Remark 4.11. We can show that (4.6) implies (4.4). Indeed, let $\phi \in \mathcal{D}\left(\mathbb{R}^{d}\right)$ and put $u=\partial_{i} \phi$. From (4.6) replaced $p$ with $p^{\prime}$,

$$
\begin{aligned}
\left\|\nabla\left(\partial_{i} \phi\right)\right\|_{L^{p^{\prime}(\cdot)}\left(\mathbb{R}^{d}\right)} & \leq C_{1}(p) \sup _{0 \neq v \in \mathcal{D}\left(\mathbb{R}^{d}\right)} \frac{\left|\left\langle\nabla\left(\partial_{i} \phi\right), \nabla v\right\rangle_{\mathbb{R}^{d}}\right|}{\|\nabla v\|_{L^{p(\cdot)}\left(\mathbb{R}^{d}\right)}} \\
& \leq C_{1}(p) \sup _{0 \neq v \in \mathcal{D}\left(\mathbb{R}^{d}\right)} \frac{\left|\left\langle\Delta \phi, \partial_{i} v\right\rangle_{\mathbb{R}^{d}}\right|}{\|\nabla v\|_{L^{p(\cdot)}\left(\mathbb{R}^{d}\right)}} \\
& \leq 2 C_{1}(p)\|\Delta \phi\|_{L^{p^{\prime}(\cdot)}\left(\mathbb{R}^{d}\right)} .
\end{aligned}
$$

Next we consider the case where $G$ is a half-space or a bended half-space. Let $\omega$ be a $C^{1}$-function defined on $\mathbb{R}^{d-1}, H=\left\{x=\left(x^{\prime}, x_{d}\right) ; x^{\prime}=\left(x_{1}, \ldots, x_{d-1}\right) \in \mathbb{R}^{d-1}, x_{d}<0\right\}$ and $H_{\omega}=\left\{x=\left(x^{\prime}, x_{d}\right) ; x_{d}<\right.$ $\left.\omega\left(x^{\prime}\right)\right\}$.

Lemma 4.12. Let $\omega$ be a $C^{1}$-function defined on $\mathbb{R}^{d-1}$ with $\left\|\nabla^{\prime} \omega\right\|_{L^{\infty}\left(\mathbb{R}^{d-1}\right)}<\infty$, where $\nabla^{\prime}=$ $\left(\partial_{1}, \ldots, \partial_{d-1}\right)$, and let $p \in \mathcal{P}_{+}^{\log }\left(\overline{H_{\omega}}\right)$. Then we have $\widehat{W}_{\bullet}^{1, p(\cdot)}\left(H_{\omega}\right)=\widehat{W}_{0}^{1, p(\cdot)}\left(H_{\omega}\right)$.

Proof. Step 1. Let $0<\rho<\infty, 0<R<\infty$ and put

$$
Z_{\rho, R}^{\omega}=\left\{x=\left(x^{\prime}, x_{d}\right) \in \mathbb{R}^{d} ;\left|x^{\prime}\right|<\rho,-R<x_{d}<\omega\left(x^{\prime}\right)\right\} .
$$

Assume $u \in \widehat{W}_{\bullet}^{1, p(\cdot)}\left(H_{\omega}\right)$. Then since $\left.u\right|_{\partial H_{\omega}}=0$, it follows from the generalized Poincaré ineequality (Theorem 2.9) that there exists a constant $C=C\left(d, C_{\log }(p)\right)$ such that

$$
\|u\|_{L^{p(\cdot)}\left(Z_{\rho R}^{\omega}\right)} \leq C(\rho+R)\|\nabla u\|_{L^{p(\cdot)}\left(Z_{\rho, R}^{\omega}\right)} .
$$


Since $\left|\omega\left(x^{\prime}\right)-\omega(0)\right| \leq\left\|\nabla^{\prime} \omega\right\|_{L^{\infty}\left(\mathbb{R}^{d-1}\right)}\left|x^{\prime}\right| \leq\left\|\nabla^{\prime} \omega\right\|_{L^{\infty}\left(\mathbb{R}^{d-1}\right)} \rho$ for $\left|x^{\prime}\right|<\rho$.

Hence $\left|\omega\left(x^{\prime}\right)\right| \leq\left\|\nabla^{\prime} \omega\right\|_{L^{\infty}\left(\mathbb{R}^{d-1}\right)} \rho+\omega_{0}$, where $\omega_{0}=|\omega(0)|$.

Step 2. Choose $\tau \in C_{0}^{\infty}\left(\mathbb{R}^{d}\right)$ such that $0 \leq \tau \leq 1$ and $\tau(x)=1$ for $|x| \leq 1$ and $\tau(x)=0$ for $|x| \geq 2$, and for $k \in \mathbb{N}$, put $\tau_{k}(x)=\tau\left(k^{-1} x\right)$. Then $\left|\nabla \tau_{k}(x)\right| \leq k^{-1}\|\nabla \tau\|_{L^{\infty}\left(\mathbb{R}^{d}\right)}$, and $\operatorname{supp}\left(\nabla \tau_{k}\right) \subset$ $A_{k}:=\left\{x \in \mathbb{R}^{d} ; k<|x|<2 k\right\}$. Put $\rho_{k}=2 k, R_{k}=2 k\left(\left\|\nabla^{\prime} \omega\right\|_{L^{\infty}\left(\mathbb{R}^{d-1}\right)}+1\right)+\omega_{0}$ and $Z_{k}=Z_{\rho_{k}, R_{k}}^{\omega}$. If $x \in H_{\omega} \cap B_{2 k}$, then $\left|x^{\prime}\right|<2 k$ and $-2 k<x_{d}<\omega\left(x^{\prime}\right)$. Hence

$$
\omega\left(x^{\prime}\right)-R_{k} \leq\left\|\nabla^{\prime} \omega\right\|_{L^{\infty}\left(\mathbb{R}^{d-1}\right)} 2 k+\omega_{0}-R_{k}=-2 k<x_{d}<\omega\left(x^{\prime}\right) .
$$

Therefore, $H_{\omega} \cap B_{2 k} \subset Z_{k}$. From (4.9), we have

$$
\begin{aligned}
\left\|u \nabla \tau_{k}\right\|_{L^{p(\cdot)}\left(H_{\omega}\right)} & \leq k^{-1}\left\|\nabla^{\prime} \omega\right\|_{L^{\infty}\left(\mathbb{R}^{d-1}\right)}\|u\|_{L^{p(\cdot)}\left(H_{\omega} \cap B_{2 k}\right)} \\
& \leq k^{-1}\left\|\nabla^{\prime} \omega\right\|_{L^{\infty}\left(\mathbb{R}^{d-1}\right)}\|u\|_{L^{p(\cdot)}\left(Z_{k}\right)} \\
& \leq C k^{-1}\left\|\nabla^{\prime} \omega\right\|_{L^{\infty}\left(\mathbb{R}^{d-1}\right)}\left(\rho_{k}+R_{k}\right)\|\nabla u\|_{L^{p(\cdot)}\left(H_{\omega}\right)} \\
& \leq C_{1}\|\nabla u\|_{L^{p(\cdot)}\left(H_{\omega}\right)},
\end{aligned}
$$

where $C_{1}$ is a constant independent of $k$. By definition of $\widehat{W}_{\bullet}^{1, p(\cdot)}\left(H_{\omega}\right), \tau_{k} u \in W_{0}^{1, p(\cdot)}\left(H_{\omega}\right) \subset$ $\widehat{W}_{0}^{1, p(\cdot)}\left(H_{\omega}\right)$.

Step 3. Let $F^{\prime} \in\left(\widehat{W}_{\bullet}^{1, p(\cdot)}\left(H_{\omega}\right)\right)^{\prime}$, that is,

$$
\left|F^{\prime}(\phi)\right| \leq\left\|F^{\prime}\right\|_{\left(\widehat{W}_{\bullet}^{1, p(\cdot)}\left(H_{\omega}\right)\right)^{\prime}}\|\nabla \phi\|_{L^{p(\cdot)}\left(H_{\omega}\right)} \text { for all } \phi \in \widehat{W}^{1, p(\cdot)}\left(H_{\omega}\right)
$$

Since $\widehat{W}_{\bullet}^{1, p(\cdot)}\left(H_{\omega}\right)$ is complete and $E_{0}^{p(\cdot)}\left(H_{\omega}\right)$ is a closed subspace of $L^{p(\cdot)}\left(H_{\omega}\right)$, we can regard $F^{\prime}$ as a continuous linear functional on $E_{0}^{p(\cdot)}\left(H_{\omega}\right)$. By the Hahn-Banach theorem, $F^{\prime}$ may be extended to a functional $\widetilde{F}^{\prime} \in\left(\boldsymbol{L}^{p(\cdot)}\left(H_{\omega}\right)\right)^{\prime}$ which is norm-preserving. Hence there exists $\boldsymbol{f} \in \boldsymbol{L}^{p^{\prime}(\cdot)}\left(H_{\omega}\right)$ such that $\|\boldsymbol{f}\|_{L^{p^{\prime}(\cdot)}\left(H_{\omega}\right)}=\left\|\widetilde{F}^{\prime}\right\|_{\left(L^{p(\cdot)}\left(H_{\omega}\right)\right)^{\prime}}=\|F\|_{\left(\widehat{W}_{\bullet}^{1, p(\cdot)}\left(H_{\omega}\right)\right)^{\prime}}$ and

$$
F^{\prime}(\phi)=\widetilde{F}^{\prime}(\phi)=\langle\boldsymbol{f}, \nabla \phi\rangle_{H_{\omega}} \text { for all } \phi \in \widehat{W}_{\bullet}^{1, p(\cdot)}\left(H_{\omega}\right) .
$$

Then

$$
F^{\prime}(u)-F^{\prime}\left(\tau_{k} u\right)=\left\langle\left(1-\tau_{k}\right) \boldsymbol{f}, \nabla u\right\rangle_{H_{\omega}}-\left\langle\boldsymbol{f}, u \nabla \tau_{k}\right\rangle_{H_{\omega}} .
$$

We have

$$
\left|\left\langle\left(1-\tau_{k}\right) \boldsymbol{f}, \nabla u\right\rangle_{H_{\omega}}\right| \leq 2\left\|\left(1-\tau_{k}\right) \boldsymbol{f}\right\|_{L^{p^{\prime}(\cdot)}\left(H_{\omega}\right)}\|\nabla u\|_{L^{p(\cdot)}\left(H_{\omega}\right)}
$$

By the Lebesgue dominated convergence theorem, we have

$$
\rho_{p^{\prime}(\cdot), H_{\omega}}\left(\left(1-\tau_{k}\right) \boldsymbol{f}\right)=\int_{H_{\omega}}\left|\left(1-\tau_{k}\right) \boldsymbol{f}\right|^{p^{\prime}(x)} d x \rightarrow 0 \text { as } k \rightarrow \infty .
$$

So it follows from Proposition 2.1 that $\left\|\left(1-\tau_{k}\right) \boldsymbol{f}\right\|_{L^{p^{\prime}(\cdot)\left(H_{\omega}\right)}} \rightarrow 0$ as $k \rightarrow \infty$. By (4.10) and $\operatorname{supp}\left(\nabla \tau_{k}\right) \subset A_{k}$,

$$
\left|\left\langle\boldsymbol{f}, u \nabla \tau_{k}\right\rangle_{H_{\omega}}\right| \leq 2 C_{1}\|\boldsymbol{f}\|_{L^{p^{\prime}(\cdot)}\left(H_{\omega} \cap A_{k}\right)}\|\boldsymbol{\nabla} u\|_{L^{p(\cdot)}\left(H_{\omega}\right)} \rightarrow 0 \text { as } k \rightarrow \infty .
$$


Hence $F^{\prime}\left(\tau_{k} u\right) \rightarrow F^{\prime}(u)$ as $k \rightarrow \infty$. Since $F^{\prime} \in\left(\widehat{W}_{\bullet}^{1, p(\cdot)}\left(H_{\omega}\right)\right)^{\prime}$ is arbitrary, we see that $\tau_{k} u \rightarrow u$ weakly in $\widehat{W}_{\bullet}^{1, p(\cdot)}\left(H_{\omega}\right)$. By Step 2, $\tau_{k} u \in \widehat{W}_{0}^{1, p(\cdot)}\left(H_{\omega}\right)$. Since $\widehat{W}_{0}^{1, p(\cdot)}\left(H_{\omega}\right)$ is a closed subspace of $\widehat{W}_{\bullet}^{1, p(\cdot)}\left(H_{\omega}\right)$ (Theorem 4.4), it is weakly closed. Therefore $u \in \widehat{W}_{0}^{1, p(\cdot)}\left(H_{\omega}\right)$.

Lemma 4.13. Let $p \in \mathcal{P}_{+}^{\log }(\bar{H})$. For $x \in \mathbb{R}^{d}$, define

$$
\widetilde{p}(x)= \begin{cases}p(x) & \text { for } x_{d} \leq 0 \\ p\left(x^{\prime},-x_{d}\right) & \text { for } x_{d}>0\end{cases}
$$

Then clearly $\widetilde{p} \in \mathcal{P}_{+}^{\log }\left(\mathbb{R}^{d}\right)$. For $u \in \widehat{W}_{\bullet}^{1, p(\cdot)}(H)$, define

$$
u_{1}(x)= \begin{cases}u(x) & \text { for } x_{d}<0 \\ 0 & \text { for } x_{d}=0 \\ -u\left(x^{\prime},-x_{d}\right) & \text { for } x_{d}>0\end{cases}
$$

Then $u_{1} \in W_{\text {loc }}^{1, \widetilde{p}(\cdot)}\left(\mathbb{R}^{d}\right), \nabla u_{1} \in L^{\widetilde{p}(\cdot)}\left(\mathbb{R}^{d}\right)$, and furthermore,

$$
\partial_{i} u_{1}(x)= \begin{cases}\left(\partial_{i} u\right)(x) & \text { for } x_{d}<0 \\ 0 & \text { for } x_{d}=0 \\ -\left(\partial_{i} u\right)\left(x^{\prime},-x_{d}\right) & \text { for } x_{d}>0\end{cases}
$$

for $i=1, \ldots, d-1$ and

$$
\partial_{d} u_{1}(x)= \begin{cases}\left(\partial_{d} u\right)(x) & \text { for } x_{d}<0 \\ \left(\partial_{d} u\right)\left(x^{\prime},-x_{d}\right) & \text { for } x_{d}>0\end{cases}
$$

In addition,

$$
\|\nabla u\|_{L^{p(\cdot)}(H)} \leq\left\|\nabla u_{1}\right\|_{L^{\tilde{p}}\left(\mathbb{R}^{d}\right)} \leq 2\|\nabla u\|_{L^{p(\cdot)}(H)} .
$$

For $\phi \in \mathcal{D}\left(\mathbb{R}^{d}\right)$, let $\left(T_{1} \phi\right)(x)=\phi(x)-\phi\left(x^{\prime},-x_{d}\right)$ for $x \in H$. Then $T_{1} \phi \in \widehat{W}_{0}^{1, p(\cdot)}(H) \cap C^{1}(H)$, $\left(T_{1} \phi\right)\left(x^{\prime}, 0\right)=0$ and there exists $R=R(\phi)>0$ such that $\left(T_{1} \phi\right)(x)=0$ for $|x|>R$ and

$$
\left\|\nabla\left(T_{1} \phi\right)\right\|_{L^{p(\cdot)}(H)} \leq 2\|\nabla \phi\|_{L^{\widetilde{p}(\cdot)}\left(\mathbb{R}^{d}\right)} .
$$

Furthermore, for $u \in \widehat{W}_{0}^{1, p(\cdot)}(H)$ and $\phi \in \mathcal{D}\left(\mathbb{R}^{d}\right)$,

$$
\left\langle\nabla u_{1}, \nabla \phi\right\rangle_{\mathbb{R}^{d}}=\left\langle\nabla u, \nabla\left(T_{1} \phi\right)\right\rangle_{H}
$$

Since this lemma follows from elementary calculations (cf. [25, Lemma 2.3]). we omit the proof.

Lemma 4.14. Let $p, q \in \mathcal{P}_{+}^{\log }(\bar{H})$. If $u \in \widehat{W}_{0}^{1, q(\cdot)}(H)$ satisfies

$$
S_{p}(u):=\sup _{0 \neq \phi \in C_{0}^{\infty}(H)} \frac{\left|\langle\nabla u, \nabla \phi\rangle_{H}\right|}{\|\nabla \phi\|_{L^{p^{\prime}(\cdot)}(H)}}<\infty,
$$

then $u \in \widehat{W}_{0}^{1, p(\cdot)}(H)$ and

$$
\|\nabla u\|_{L^{p(\cdot)}(H)} \leq C_{2}(p) S_{p}(u),
$$

where $C_{2}(p)=2 C_{1}>0, C_{1}$ is a constant as in (4.6). 
Proof. For any function $\phi \in \mathcal{D}\left(\mathbb{R}^{d}\right)$, we consider $T_{1} \phi$. If $\operatorname{supp} \phi \subset B_{R}$, then $\operatorname{supp} T_{1} \phi \subset B_{R}$, $\left(T_{1} \phi\right)\left(x^{\prime}, 0\right)=0$ and $\nabla\left(T_{1} \phi\right) \in L^{\infty}(H)$. Choose $\eta \in C^{\infty}\left(\mathbb{R}^{d}\right)$ such that $0 \leq \eta \leq 1$ and $\eta(x)=1$ for $|x| \geq 1$ and $\eta(x)=0$ for $|x| \leq 1 / 2$. For $k \in \mathbb{N}$, put $\eta_{k}(x)=\eta(k x)$ and $\phi_{k}(x)=\eta_{k}(x)\left(T_{1} \phi\right)(x)$. Then for $s=q^{\prime}$ and $s=p^{\prime}$,

$$
\left\|\nabla\left(T_{1} \phi\right)-\nabla \phi_{k}\right\|_{L^{s(\cdot)}(H)} \leq\left\|\left(1-\eta_{k}(x)\right) \nabla\left(T_{1} \phi\right)\right\|_{L^{s(\cdot)}(H)}+\left\|\left(\nabla \eta_{k}(x)\right) T_{1} \phi\right\|_{L^{s(\cdot)}(H)} .
$$

Here from the Lebesgue dominated convergence theorem,

$$
\left\|\left(1-\eta_{k}(x)\right) \nabla\left(T_{1} \phi\right)\right\|_{L^{s(\cdot)}(H)} \rightarrow 0 \text { as } k \rightarrow \infty .
$$

Since supp $\eta_{k} \subset\left\{x \in \mathbb{R}^{d} ; 1 /(2 k)<|x|<1 / k\right\}=: A_{k}$, It follows from the Poincaré inequality (Theorem 2.9) that

$$
\begin{aligned}
& \left\|\left(\nabla \eta_{k}(x)\right) T_{1} \phi\right\|_{L^{s(\cdot)}(H)} \leq k\|\nabla \eta\|_{L^{\infty}\left(\mathbb{R}^{d}\right)}\left\|T_{1} \phi\right\|_{L^{s(\cdot)}\left(H \cap A_{k}\right)} \\
& \quad \leq k\|\nabla \eta\|_{L^{\infty}\left(\mathbb{R}^{d}\right)} \frac{1}{k}\left\|\nabla\left(T_{1} \phi\right)\right\|_{L^{s(\cdot)}\left(H \cap A_{k}\right)}=\|\nabla \eta\|_{L^{\infty}\left(\mathbb{R}^{d}\right)}\left\|\nabla\left(T_{1} \phi\right)\right\|_{L^{s(\cdot)}\left(H \cap A_{k}\right)} \rightarrow 0
\end{aligned}
$$

as $k \rightarrow \infty$. Therefore, since $u \in \widehat{W}_{0}^{1, q(\cdot)}(H)$, we have

$$
S_{p}(u) \geq \frac{\left|\left\langle\nabla u, \nabla \phi_{k}\right\rangle_{H}\right|}{\left\|\boldsymbol{\nabla} \phi_{k}\right\|_{L^{p^{\prime}(\cdot)}(H)}} \rightarrow \frac{\left|\left\langle\nabla u, \nabla\left(T_{1} \phi\right)\right\rangle_{H}\right|}{\left\|\nabla\left(T_{1} \phi\right)\right\|_{L^{p^{\prime}(\cdot)}(H)}} .
$$

Hence for $0 \neq \phi \in C_{0}^{\infty}\left(\mathbb{R}^{d}\right)$ such that $T_{1} \phi \neq 0$, by Lemma 4.13

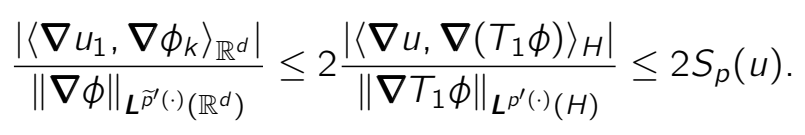

By Lemma 4.10, we see that $\nabla u_{1} \in L^{\widetilde{p}}\left(\mathbb{R}^{d}\right)$, so $\nabla u \in L^{p(\cdot)}(H)$. Since $u \in \widehat{W}_{0}^{1, q(\cdot)}(H)$, we can see that $u \in \widehat{W}_{0}^{1, p(\cdot)}(H)$ as in the proof of Lemma 4.12, and

$$
\|\nabla u\|_{L^{p(\cdot)}(H)} \leq\left\|\nabla u_{1}\right\|_{L^{\widetilde{p}(\cdot)}\left(\mathbb{R}^{d}\right)} \leq 2 C_{1} S_{p}(u) .
$$

Lemma 4.15. Let $\omega$ be a $C^{1}$-function on $\mathbb{R}^{d-1}$ such that there exists $R=R(\omega)>0$ such that $\omega\left(x^{\prime}\right)=0$ for $\left|x^{\prime}\right|>R$ and let $p \in \mathcal{P}_{+}^{\log }\left(\overline{H_{\omega}}\right)$. Assume that there exists a constant $K_{p}=K(p, d)>0$ such that

$$
\left\|\nabla^{\prime} \omega\right\|_{L^{\infty}\left(\mathbb{R}^{d-1}\right)} \leq K_{p} .
$$

Then there exists a constant $C_{3}(s)=C_{3}\left(s, d, K_{p}\right)>0$ such that for all $u \in \widehat{W}_{0}^{1, s(\cdot)}\left(H_{\omega}\right)$,

$$
\|\nabla u\|_{L^{s(\cdot)}\left(H_{\omega}\right)} \leq C_{3}(s) \sup _{0 \neq \phi \in C_{0}^{\infty}\left(H_{\omega}\right)} \frac{\left|\langle\nabla u, \nabla \phi\rangle_{H_{\omega}}\right|}{\|\nabla \phi\|_{L^{s^{\prime}(\cdot)}\left(H_{\omega}\right)}}
$$

for $s=p, p^{\prime}$. 
Proof. Let $y: \mathbb{R}^{d} \rightarrow \mathbb{R}^{d}$ be a map defined by

$$
\begin{cases}y_{i}(x)=x_{i} & \text { for } i=1, \ldots, d-1 \\ y_{d}(x)=x_{d}-\omega\left(x^{\prime}\right) & \end{cases}
$$

Then $y$ is a $C^{1}$-map and bijective, $y\left(H_{\omega}\right)=H, y\left(\partial H_{\omega}\right)=\partial H$ and the Jacobian $J(y(x))=1$ for $x \in \mathbb{R}^{d}$. The inverse map $x: \mathbb{R}^{d} \rightarrow \mathbb{R}^{d}$ is given by

$$
\left\{\begin{array}{l}
x_{i}(y)=y_{i} \\
x_{d}(y)=y_{d}+\omega\left(y^{\prime}\right)
\end{array}\right.
$$

For $s \in \mathcal{P}_{+}^{\log }\left(\overline{H_{\omega}}\right)$, define $\widetilde{s}(y)=s(x(y))$. Then $\widetilde{s} \in \mathcal{P}_{+}^{\log }(\bar{H})$. If $u \in \widehat{W}_{0}^{1, s(\cdot)}\left(H_{\omega}\right)$ and define $\widetilde{u}(y)=u(x(y))$ for $y \in H$, then $\widetilde{u} \in \widehat{W}_{0}^{1, \widetilde{s}(\cdot)}(H)$. Conversely, if $\widetilde{u} \in \widehat{W}_{0}^{1, \widetilde{s}(\cdot)}(H)$, then $u(x)=\widetilde{u}(y(x))$ for $x \in H_{\omega}$ belongs to $\widehat{W}_{0}^{1, s(\cdot)}\left(H_{\omega}\right)$. Since

$$
\left\{\begin{array}{l}
\partial_{i} u(x)=\left(\partial_{i} \widetilde{u}\right)(y(x))-\left(\partial_{d} \widetilde{u}\right)(y(x)) \partial_{i} \omega\left(x^{\prime}\right) \quad \text { for } i=1, \ldots, d-1, \\
\partial_{d} u(x)=\left(\partial_{d} \widetilde{u}\right)(y(x))
\end{array}\right.
$$

there exists a constant $d_{1}(s)=d_{1}(s, d)>0$ such that

$$
\begin{aligned}
\|\nabla u\|_{L^{s(\cdot)}\left(H_{\omega}\right)} & \leq d_{1}(s)\left(1+\left\|\nabla^{\prime} \omega\right\|_{L^{\infty}\left(\mathbb{R}^{d-1}\right)}\right)\|\nabla \widetilde{u}\|_{L^{\widetilde{s}(\cdot)}(H)}, \\
\|\nabla \widetilde{u}\|_{L^{\widetilde{s}(\cdot)}(H)} & \leq d_{1}(s)\left(1+\left\|\nabla^{\prime} \omega\right\|_{L^{\infty}\left(\mathbb{R}^{d-1}\right)}\right)\|\nabla u\|_{L^{s(\cdot)}\left(H_{\omega}\right)} .
\end{aligned}
$$

Thus the map $\widehat{W}_{0}^{1, s(\cdot)}\left(H_{\omega}\right) \ni u \mapsto \widetilde{u} \in \widehat{W}_{0}^{1, \widetilde{s}(\cdot)}(H)$ is continuous, linear and bijective.

Let $u \in \widehat{W}_{0}^{1, s(\cdot)}\left(H_{\omega}\right)$ and $\phi \in \widehat{W}_{0}^{1, s^{\prime}(\cdot)}\left(H_{\omega}\right)$. By elementary calculations, we have

$$
\langle\nabla u, \nabla \phi\rangle_{H_{\omega}}=\int_{H_{\omega}} \nabla u(x) \cdot \nabla \phi(x) d x=\int_{H}(\nabla \widetilde{u})(y) \cdot(\nabla \widetilde{\phi})(y) d y-B_{\omega}[\nabla \widetilde{u}, \nabla \widetilde{\phi}],
$$

where

$$
B_{\omega}[\nabla \widetilde{u}, \nabla \widetilde{\phi}]=-\sum_{i=1}^{d-1} \int_{H}\left(\left(\partial_{d} \widetilde{u}\right)\left(\partial_{i} \widetilde{\phi}\right)+\left(\partial_{i} \widetilde{u}\right)\left(\partial_{d} \widetilde{\phi}\right) \partial_{i} \omega\left(y^{\prime}\right)\right) d y+\int_{H}\left(\partial_{d} \widetilde{u}\right)\left(\partial_{d} \widetilde{\phi}\right)|\nabla \omega|^{2} d y .
$$

By the generalized Hölder inequality, there exists a constant $d_{2}(s)>0$ such that

$$
\left|B_{\omega}[\nabla \widetilde{u}, \nabla \widetilde{\phi}]\right| \leq d_{2}(s)\left\|\nabla^{\prime} \omega\right\|_{L^{\infty}\left(\mathbb{R}^{d-1}\right)}\left(1+\left\|\nabla^{\prime} \omega\right\|_{L^{\infty}\left(\mathbb{R}^{d-1}\right)}\right)\|\nabla \widetilde{u}\|_{L^{\widetilde{s}(\cdot)}(H)}\|\nabla \widetilde{\phi}\|_{L^{\tilde{s}^{(} \cdot(\cdot)}(H)} .
$$

Therefore, for $0 \neq \phi \in \widehat{W}_{0}^{1, s^{\prime}(\cdot)}\left(H_{\omega}\right)$,

$$
\begin{array}{r}
\frac{\left|\langle\nabla u, \nabla \phi\rangle_{H_{\omega}}\right|}{\|\nabla \phi\|_{L^{s^{\prime}(\cdot)}\left(H_{\omega}\right)}} \geq\left(d_{1}\left(s^{\prime}\right)\left(1+\left\|\nabla^{\prime} \omega\right\|_{L^{\infty}\left(\mathbb{R}^{d-1}\right)}\right)\right)^{-1}\left\{\frac{\left|\langle\nabla \widetilde{u}, \nabla \widetilde{\phi}\rangle_{H}\right|}{\|\nabla \widetilde{\phi}\|_{L^{\widetilde{s}^{\prime} \cdot(\cdot)}(H)}}\right. \\
\left.-d_{2}(s)\left\|\nabla^{\prime} \omega\right\|_{L^{\infty}\left(\mathbb{R}^{d-1}\right)}\left(1+\left\|\nabla^{\prime} \omega\right\|_{L^{\infty}\left(\mathbb{R}^{d-1}\right)}\right)\|\nabla \widetilde{u}\|_{L^{\widetilde{s}(\cdot)}(H)}\right\} .
\end{array}
$$

Define

$$
K_{p}=\min \left\{\frac{1}{2}, \min \left\{\left(4 C_{2}(s) d_{2}(s)\right)^{-1} ; s=p, p^{\prime}\right\}\right\}
$$


where $C_{2}(s)>0$ is a constant defined in Lemma 4.14. If $\left\|\nabla^{\prime} \omega\right\|_{L^{\infty}\left(\mathbb{R}^{d-1}\right)} \leq K_{p}$, then

$$
\begin{aligned}
& \sup _{0 \neq \phi \in \widehat{W}_{0}^{1, s^{\prime}(\cdot)}\left(H_{\omega}\right)} \frac{\left|\langle\nabla u, \nabla \phi\rangle_{H_{\omega}}\right|}{\|\boldsymbol{\nabla} \phi\|_{L^{s^{\prime}(\cdot)}\left(H_{\omega}\right)}} \geq\left(2 d_{1}\left(s^{\prime}\right)\right)^{-1}\left\{\sup _{0 \neq \widetilde{\phi} \in \widehat{W}^{1, \widetilde{s}^{\prime}(\cdot)}(H)} \frac{\left|\langle\boldsymbol{\nabla} \widetilde{u}, \boldsymbol{\nabla} \widetilde{\phi}\rangle_{H}\right|}{\|\nabla \widetilde{\phi}\|_{L^{s^{\prime}(\cdot)}(H)}}\right. \\
& -2 d_{2}(s)\left(\left(4 C_{2}(s) d_{2}(s)\right)^{-1}\|\nabla \widetilde{u}\|_{L^{\tilde{s}(\cdot)}(H)}\right\} \\
& \geq\left(4 d_{s}\left(s^{\prime}\right) C_{s}(s)\right)^{-1}\|\nabla \widetilde{u}\|_{L^{\widetilde{s}(\cdot)}(H)} \geq C_{3}(s)\|\nabla u\|_{L^{s(\cdot)}\left(H_{\omega}\right)},
\end{aligned}
$$

where $C_{3}(s)=\left(8 d_{s}(s) d_{1}\left(s^{\prime}\right) C_{2}(s)\right)^{-1}$

Lemma 4.16. Suppose $(G A)$. Let $x_{0} \in G$ and $B_{R}\left(x_{0}\right) \Subset G$, and let $p \in \mathcal{P}_{+}^{\log }\left(\mathbb{R}^{d}\right)$. Then for $0<R^{\prime}<R$, there exists a constant $C_{3}\left(p, R, R^{\prime}\right)>0$ such that

$$
\|\nabla(\eta u)\|_{L^{p(\cdot)}(G)} \leq C_{3}\left(p, R, R^{\prime}\right) \sup _{0 \neq v \in C_{0}^{\infty}\left(B_{R}\left(x_{0}\right)\right)} \frac{\left|\langle\nabla(\eta u), \nabla v\rangle_{G}\right|}{\|\nabla v\|_{L^{p^{\prime}(\cdot)}\left(B_{R}\left(x_{0}\right)\right)}}
$$

for all $u \in \widehat{W}_{\bullet}^{1, p(\cdot)}(G)$ and $\eta \in C_{0}^{\infty}\left(B_{R^{\prime}}\left(x_{0}\right)\right)$.

Proof. Let $\rho \in \mathcal{D}\left(B_{R}\left(x_{0}\right)\right)$ such that $0 \leq \rho \leq 1$ and $\rho(x)=1$ for $x \in B_{R^{\prime}}\left(x_{0}\right)$. If $\phi \in \mathcal{D}\left(\mathbb{R}^{d}\right)$, put $c_{\phi}=\frac{1}{\left|B_{R}\left(x_{0}\right)\right|} \int_{B_{R}\left(x_{0}\right)} \phi d x$ and $v=\rho\left(\phi-c_{\phi}\right)$. By the Poincaré inequlity (Theorem 2.7),

$$
\left\|\phi-c_{\phi}\right\|_{\left.L^{p^{\prime}(\cdot)} B_{R^{\prime}}\left(x_{0}\right)\right)} \leq C_{R}\|\nabla v\|_{L^{p^{\prime}(\cdot)}\left(B_{R}\left(x_{0}\right)\right)} .
$$

Here we have

$$
\|\nabla v\|_{L^{p^{\prime}(\cdot)\left(B_{R}\left(x_{0}\right)\right)}} \leq\left(1+C_{R}\|\nabla \rho\|_{L^{\infty}\left(B_{R}\left(x_{0}\right)\right)}\right)\|\nabla \phi\|_{L^{p^{\prime}(\cdot)\left(\mathbb{R}^{d}\right)}} .
$$

Since $\nabla \rho=0$ on $B_{R^{\prime}}\left(x_{0}\right), \rho=1$ on $B_{R^{\prime}}\left(x_{0}\right)$ and $\nabla v=(\nabla \rho)\left(\phi-c_{\phi}\right)+\rho \boldsymbol{\nabla} \phi$, we see that $\nabla v=\nabla \phi$ on $B_{R^{\prime}}\left(x_{0}\right)$. If $\phi \neq 0$ and $v \neq 0$, then we have

$$
\frac{\left|\langle\nabla(\eta u), \nabla \phi\rangle_{\mathbb{R}^{d}}\right|}{\|\nabla \phi\|_{L^{p^{\prime}(\cdot)}\left(\mathbb{R}^{d}\right)}} \leq\left(1+C_{R}\|\rho\|_{L^{\infty}\left(B_{R}\left(x_{0}\right)\right)}\right) \frac{\left|\langle\nabla(\eta u), \nabla \phi\rangle_{G}\right|}{\|\nabla v\|_{L^{p^{\prime}(\cdot)}\left(B_{R}\left(x_{0}\right)\right)}} .
$$

By Lemma 4.10, we can see that

$$
\begin{aligned}
\|\nabla(\eta u)\|_{L^{p(\cdot)}(G)} & \leq C_{1}(p) \sup _{0 \neq \phi \in C_{0}^{\infty}\left(\mathbb{R}^{d}\right)} \frac{\left|\langle\nabla(\eta u), \nabla \phi\rangle_{\mathbb{R}^{d}}\right|}{\|\nabla \phi\|_{L^{p^{\prime}(\cdot)}\left(\mathbb{R}^{d}\right)}} \\
& \leq C_{1}(p)\left(1+C_{R}\|\nabla \rho\|_{L^{\infty}\left(\mathbb{R}^{d}\right)}\right) \sup _{0 \neq v \in C_{0}^{\infty}\left(B_{R}\left(x_{0}\right)\right)} \frac{\left|\langle\nabla(\eta u), \nabla v\rangle_{G}\right|}{\|\nabla v\|_{L^{p^{\prime}(\cdot)}\left(B_{R}\left(x_{0}\right)\right)}}
\end{aligned}
$$

Lemma 4.17. Suppose $(G A)$ and $p \in \mathcal{P}_{+}^{\log }(\bar{G})$. For each $x_{0} \in \partial G$, there exist $R=R\left(p, x_{0}, \partial G\right)>0$ and a constant $C_{5}=C_{5}(R)>0$ such that

$$
\|\nabla(\eta u)\|_{L^{p(\cdot)}(G)} \leq C_{5} \sup _{0 \neq v \in \widehat{W}^{1, p^{\prime}(\cdot)}\left(G_{R}\left(x_{0}\right)\right)} \frac{\left|\langle\nabla(\eta u), \nabla v\rangle_{G}\right|}{\|\nabla v\|_{L^{p^{\prime}(\cdot)}\left(G_{R}\left(x_{0}\right)\right)}}
$$

for all $u \in \widehat{W}_{\bullet}^{1, p(\cdot)}(G)$ and $\eta \in C_{0}^{\infty}\left(B_{R / 2}\left(x_{0}\right)\right)$. 
Proof. There exist $\rho>0$ and a $C^{1}$-function $\sigma$ on $\overline{B_{\rho}\left(x_{0}\right)}$ with $(\nabla \sigma)\left(x_{0}\right) \neq 0$ such that $G \cap B_{\rho}\left(x_{0}\right)=$ $\left\{x \in B_{\rho}\left(x_{0}\right) ; \sigma(x)<0\right\}$ and $\partial G \cap B_{R}\left(x_{0}\right)=\left\{x \in B_{\rho}\left(x_{0}\right) ; \sigma(x)=0\right\}$. Then $\left|\nabla \sigma\left(x_{0}\right)\right|^{-1} \nabla \sigma\left(x_{0}\right)$ is the unit outer normal vector at $x_{0}$. Hence there exists an orthogonal matrix $S$ such that $S\left(\left|\nabla \sigma\left(x_{0}\right)\right|^{-1} \nabla \sigma\left(x_{0}\right)\right)=e_{d}=(0, \ldots, 0,1)^{t}$. Define a transformation $y=y(x)=S\left(x-x_{0}\right)$. Then $y: B_{\rho}\left(x_{0}\right) \rightarrow \widehat{B}_{\rho}(0)=\left\{y \in \mathbb{R}^{d} ;|y|<\rho\right\}$ is a $C^{1}$-bijective mapping and define $\widehat{\sigma}(y)=$ $\sigma\left(x_{0}+S^{-1} y\right)=\sigma(x)$. Hence $\left(\nabla_{y} \widehat{\sigma}\right)(0)=S\left(\nabla_{x} \sigma\right)\left(x_{0}\right)=\left|\nabla \sigma\left(x_{0}\right)\right| \boldsymbol{e}_{d}$, so $\left(\nabla_{y^{\prime}} \widehat{\sigma}\right)(0) \neq 0$ and $\left(\partial_{y_{d}} \widehat{\sigma}\right)(0)=\left|\nabla \sigma\left(x_{0}\right)\right|>0$. By the implicit function theorem, there exist $0<\rho^{\prime}<\rho, h>0$ and $\psi \in C^{1}\left(\overline{B_{\rho^{\prime}}^{\prime}}\right)$, where $B_{\rho^{\prime}}^{\prime}=\left\{y^{\prime} \in \mathbb{R}^{d-1} ;\left|y^{\prime}\right|<\rho^{\prime}\right\}$, such that

$$
Z=Z_{\rho^{\prime}, h}=\left\{y=\left(y^{\prime}, y_{d}\right) \in \mathbb{R}^{d} ;\left|y^{\prime}\right|<\rho^{\prime},\left|y_{d}\right|<h\right\} \subset \widehat{B}_{\rho}(0)
$$

$\left(y^{\prime}, \psi\left(y^{\prime}\right)\right) \in Z$ if $y^{\prime} \in B_{\rho^{\prime}}^{\prime}$ and $\widehat{\sigma}\left(y^{\prime}, \psi\left(y^{\prime}\right)\right)=0$ for $y^{\prime} \in B_{\rho^{\prime}}^{\prime}$. Conversely, if $\left(y^{\prime}, y_{d}\right) \in Z$ and $\sigma\left(y^{\prime}, y_{d}\right)=0$, then $y_{d}=\psi\left(y^{\prime}\right), \psi(0)=0$ and $\nabla_{y^{\prime}}^{\prime} \psi(0)=0$. Then clearly, $\widehat{G} \cap Z=\left\{y=\left(y^{\prime}, y_{d}\right) \in\right.$ $\left.Z ; y_{d}<\psi\left(y^{\prime}\right)\right\}$ and $\partial \widehat{G} \cap Z=\left\{y=\left(y^{\prime}, y_{d}\right) \in Z ; y_{d}=\psi\left(y^{\prime}\right)\right\} . \quad \psi(0)=0$ and $\left(\nabla_{y^{\prime}}^{\prime} \psi\right)(0)=0$. Put $G_{\rho}=G \cap B_{\rho}\left(x_{0}\right), \widehat{G}=S G$ and $\widehat{G}_{\rho}=\widehat{G} \cap \widehat{B}_{\rho}(0)$. For $p \in \mathcal{P}_{+}^{\log }\left(G_{\rho}\right), u \in \widehat{W}_{\bullet}^{1, p(\cdot)}\left(G_{\rho}\right)$ and $v \in \widehat{W}_{0}^{1, p^{\prime}(\cdot)}\left(G_{\rho}\right)$, define $\widehat{p}(y)=p\left(x_{0}+S^{-1} y\right), \widehat{u}(y)=u\left(x_{0}+S^{-1} y\right)$ and $\widehat{v}(y)=v\left(x_{0}+S^{-1} y\right)$. Then by the elementary calculations, we have

$$
\langle\nabla \widehat{u}, \nabla \widehat{v}\rangle_{\widehat{G}_{\rho}}=\langle\nabla u, \nabla v\rangle_{G_{\rho}}
$$

and $\|\boldsymbol{\nabla} \widehat{u}\|_{L^{\widehat{p}(\cdot)}\left(\widehat{G}_{\rho}\right)}=\|\nabla u\|_{L^{p(\cdot)}\left(G_{\rho}\right)}$.

Let $\eta \in \mathcal{D}\left(\mathbb{R}^{d-1}\right)$ such that $\eta\left(y^{\prime}\right)=1$ for $\left|y^{\prime}\right| \leq 1$ and $\eta\left(y^{\prime}\right)=0$ for $\left|y^{\prime}\right| \geq 2$. For $0<\lambda<\rho^{\prime} / 2$, put $\eta_{\lambda}\left(y^{\prime}\right)=\eta\left(\lambda^{-1} y^{\prime}\right)$, and define

$$
\omega_{\lambda}\left(y^{\prime}\right)= \begin{cases}\eta_{\lambda}\left(y^{\prime}\right) \psi\left(y^{\prime}\right) & \text { for }\left|y^{\prime}\right| \leq \rho^{\prime} \\ 0 & \text { otherwise }\end{cases}
$$

Then $\nabla^{\prime} \omega_{\lambda}\left(y^{\prime}\right)=\left(\nabla^{\prime} \eta_{\lambda}\left(y^{\prime}\right)\right) \psi\left(y^{\prime}\right)+\eta_{\lambda} \nabla^{\prime} \psi\left(y^{\prime}\right)$. Since $\psi(0)=0$ and $\psi \in C^{1}\left(\overline{B_{\rho^{\prime}}^{\prime}}\right)$, using the mean value theorem,

$$
\left|\psi\left(y^{\prime}\right)\right|=\left|\psi\left(y^{\prime}\right)-\psi(0)\right| \leq\left|\left(\nabla^{\prime} \psi\right)\left(\theta y^{\prime}\right)\right|\left|y^{\prime}\right| \leq 2 \lambda\left|\left(\nabla^{\prime} \psi\right)\left(\theta y^{\prime}\right)\right| \text { for some } 0<\theta<1
$$

Hence

$$
\left|\left(\nabla^{\prime} \eta_{\lambda}\left(y^{\prime}\right)\right) \psi\left(y^{\prime}\right)\right| \leq 2 \lambda^{-1}\left|\left(\nabla^{\prime} \eta\right)\left(\lambda^{-1} y^{\prime}\right)\right| \lambda\left|\nabla^{\prime} \psi\left(\theta y^{\prime}\right)\right|=2\left|\left(\nabla^{\prime} \eta\right)\left(\lambda^{-1} y^{\prime}\right)\right|\left|\nabla^{\prime} \psi\left(\theta y^{\prime}\right)\right| .
$$

Therefore, we have

$$
\sup _{\left|y^{\prime}\right| \leq \rho}\left|\left(\nabla \eta_{\lambda}\left(y^{\prime}\right)\right) \psi\left(y^{\prime}\right)\right| \leq\left\|\nabla^{\prime} \eta\right\|_{L^{\infty}\left(\mathbb{R}^{d-1}\right)} \sup _{\left|y^{\prime}\right| \leq 2 \lambda}\left|\left(\nabla^{\prime} \psi\right)\left(y^{\prime}\right)\right| \rightarrow 0
$$

as $\lambda \rightarrow 0$ because $\nabla^{\prime} \psi$ is continuous function and $\nabla^{\prime} \psi(0)=0$. Moreover,

$$
\sup _{\left|y^{\prime}\right| \leq \rho}\left|\eta_{\lambda}\left(y^{\prime}\right) \nabla^{\prime} \psi\left(y^{\prime}\right)\right| \leq \sup _{\left|y^{\prime}\right| \leq 2 \lambda}\|\eta\|_{L^{\infty}\left(\mathbb{R}^{d-1}\right)} \|\left(\nabla^{\prime} \psi\right)\left(y^{\prime}\right) \mid \rightarrow 0 \text { as } \lambda \rightarrow 0 .
$$


Thereby, if we choose $\lambda>0$ small enough, then $\left\|\nabla^{\prime} \omega_{\lambda}\right\|_{L^{\infty}\left(\mathbb{R}^{d-1}\right)} \leq K_{p}$, where $K_{p}$ is as in Lemma 4.15. Let $R=R(\widehat{p}, 0, \partial \widehat{G})=\lambda$. Then $H_{\omega_{\lambda}} \cap B_{R}=\widehat{G} \cap B_{R}$. If $\eta \in \mathcal{D}\left(B_{R / 2}\right)$ and $u \in \widehat{W}_{\bullet}^{1, p(\cdot)}(G)$, then $\eta \widehat{u} \in W_{0}^{1, \widehat{p}}(\widehat{G})$ by definition of $\widehat{W}_{\bullet}^{1, \widehat{p}}(\widehat{G})$ and $\eta \widehat{u}$ vanishes outside $\widehat{G} \cap B_{R}=H_{\omega_{\lambda}} \cap B_{R}$. We extend $\eta \widehat{u}$ by zero to $H_{\omega_{\lambda}}$. Then $\eta \widehat{u} \in W_{0}^{1, \widehat{p}}\left(H_{\omega}\right) \subset \widehat{W}_{0}^{1, \widehat{p}}\left(H_{\omega}\right)$. By Lemma 4.15, we have

$$
\|\nabla(\eta \widehat{u})\|_{L^{\widehat{p}(\cdot)}\left(H_{\omega}\right)} \leq C_{3}(p) \sup _{0 \neq \widehat{v} \in C_{0}^{\infty}\left(H_{\omega}\right)} \frac{\left|\langle\nabla(\eta \widehat{u}), \nabla \widehat{v}\rangle_{H_{\omega}}\right|}{\|\nabla \widehat{v}\|_{L^{\widehat{p}(\cdot)}\left(H_{\omega}\right)}} .
$$

We show (4.12). Let $\rho \in C_{0}^{\infty}\left(B_{R}\right)$ such that $0 \leq \rho \leq 1$ and $\rho(x)=1$ on $B_{R / 2}$. If $\widehat{v} \in \widehat{W}_{\bullet}^{1, \widehat{p}(\cdot)}\left(H_{\omega}\right)$, then $\rho \widehat{v} \in W_{0}^{1, \widehat{p}(\cdot)}\left(H_{\omega}\right)$ and by Poincaré inequality,

$$
\begin{aligned}
\|\nabla(\rho \widehat{v})\|_{L^{\hat{p}^{\prime}(\cdot)\left(H_{\omega_{\lambda}}\right)}} & \leq\|\nabla \rho\|_{L^{\infty}\left(B_{R}\right)}\|\widehat{v}\|_{L^{\hat{p}^{\prime}(\cdot)}\left(Z_{R, R}^{\left.\omega_{\lambda}\right)}\right.}+\|\nabla \widehat{v}\|_{L^{\widehat{p}^{\prime} \cdot(\cdot)}\left(H_{\omega_{\lambda}}\right)} \\
& \leq\left(\|\nabla \rho\|_{L^{\infty}\left(B_{R}\right)} c R+1\right)\|\nabla \widehat{v}\|_{L^{\widehat{p}^{\prime}(\cdot)}\left(H_{\omega_{\lambda}}\right)} .
\end{aligned}
$$

If $\widehat{v} \neq 0$ and $\rho \widehat{v} \neq 0$, then we have

$$
\frac{\left|\langle\nabla(\eta \widehat{u}), \nabla \widehat{v}\rangle_{H_{\omega_{\lambda}}}\right|}{\left.\|\nabla \widehat{v}\|_{L^{\prime}(\cdot)\left(H_{\omega_{\lambda}}\right.}\right)} \leq\left(\|\nabla \rho\|_{L^{\infty}\left(B_{R}\right)} C R+1\right) \frac{\left|\langle\nabla(\eta \widehat{u}), \nabla(\rho \widehat{v})\rangle_{H_{\omega_{\lambda}}}\right|}{\|\nabla(\rho \widehat{v})\|_{L^{\prime}(\cdot)\left(H_{\omega_{\lambda}}\right)}} .
$$

Thus (4.12) follows from Lemma 4.15 with $C_{5}=C_{2}(p)\left(\|\nabla \rho\|_{L^{\infty}\left(B_{R}\right)} C R+1\right)$.

\section{Proof of Theorem 3.1}

First we derive the uniqueness.

Theorem 5.1. Let $G$ be a bounded domain of $\mathbb{R}^{d}(d \geq 2)$ with a $C^{1}$-boundary $\partial G$, and let $p \in \mathcal{P}_{+}^{\log }(G)$. If $u \in W_{0}^{1, p(\cdot)}(G)$ satisfies

$$
\langle\nabla u, \nabla \phi\rangle_{G}=0 \text { for all } \phi \in W_{0}^{1, p^{\prime}(\cdot)}(G)
$$

then we have $u=0$ a.e. in $G$.

Proof. Since $p(x) \geq p^{-}$for all $x \in G$ and $G$ is a bounded domain, we see that $W_{0}^{1, p(\cdot)}(G) \subset W_{0}^{1, p^{-}}(G)$. Since $\mathcal{D}(G) \subset W_{0}^{1, p^{\prime}(\cdot)}(G)$, we have $\langle\nabla u, \nabla \phi\rangle_{G}=0$ for all $\phi \in \mathcal{D}(G)$. Hence it follows from the fact that $\mathcal{D}(G)$ is dense in $W^{1,\left(p^{-}\right)^{\prime}}(G)$ that we can see that

$$
\langle\nabla u, \nabla \phi\rangle_{G}=0 \text { for all } \phi \in W_{0}^{1,\left(p^{-}\right)^{\prime}(\cdot)}(G)
$$

by continuity. Therefore by [25, Theorem 3.1], we have $u=0$ a.e. in $G$.

We give a proof of Theorem 3.1. Suppose that (3.1) does not hold. Then there exists $\left\{u_{k}\right\}_{k=1}^{\infty} \subset$ $W_{0}^{1, p(\cdot)}(G)$ such that

$$
\left\|\nabla u_{k}\right\|_{L^{p(\cdot)}(G)}=1
$$

and

$$
\varepsilon_{k}=\sup _{0 \neq \phi \in W_{0}^{1, p^{\prime}(\cdot)}(G)} \frac{\left|\left\langle\nabla u_{k}, \nabla \phi\right\rangle_{G}\right|}{\|\boldsymbol{\nabla} \phi\|_{L^{p^{\prime}(\cdot)}(G)}} \rightarrow 0 \text { as } k \rightarrow \infty
$$


By the Poincaré inequality and (5.1),

$$
\left\|u_{k}\right\|_{L^{p(\cdot)}(G)} \leq c \operatorname{diam}(G)\left\|\nabla u_{k}\right\|_{L^{p(\cdot)}(G)}=c \operatorname{diam}(G) .
$$

Hence $\left\{u_{k}\right\}_{k=1}^{\infty}$ is bounded in a reflexive Banach space $W_{0}^{1, p(\cdot)}(G)$, so passing to a subsequence (still denoted by $\left.\left\{u_{k}\right\}\right)$, we may assume that there exists $u \in W_{0}^{1, p(\cdot)}(G)$ such that $u_{k} \rightarrow u$ weakly in $W_{0}^{1, p(\cdot)}(G)$. For each $\phi \in W_{0}^{1, p^{\prime}(\cdot)}(G)$, from (5.2), we have

$$
\langle\nabla u, \nabla \phi\rangle_{G}=\lim _{k \rightarrow \infty}\left\langle\nabla u_{k}, \nabla \phi\right\rangle_{G}=0
$$

Therefore it follows from Theorem 5.1 that $u=0$. Since $G$ is bounded, the embedding $W_{0}^{1, p(\cdot)}(G) \hookrightarrow$ $L^{p(\cdot)}(G)$ is compact (cf. [11, Theorem 8.4.2], so $u_{k} \rightarrow 0$ strongly in $L^{p(\cdot)}(G)$.

By Lemma 4.17, for each $x_{0} \in \partial G$, there exist $R_{0}=R_{0}\left(p, x_{0}, \partial G\right)>0$ and $C_{5}=C_{5}\left(R_{0}\right)>0$ such that

$$
\|\nabla(\eta u)\|_{L^{p(\cdot)}(G)} \leq C_{5} \sup _{0 \neq v \in W_{0}^{1, p^{\prime}(\cdot)}\left(G \cap B_{R_{0}}\left(x_{0}\right)\right)} \frac{\left|\langle\nabla(\eta u), \nabla v\rangle_{G}\right|}{\|\nabla v\|_{L^{p^{\prime}(\cdot)}\left(G \cap B_{R_{0}}\left(x_{0}\right)\right)}}
$$

for all $u \in W_{0}^{1, p(\cdot)}(G)$ and $\left.\eta \in C_{0}^{\infty}\left(B_{R_{0} / 2}\right)\left(x_{0}\right)\right)$. Since $\partial G$ is compact, there exist finitely many $x_{i} \in \partial G(i=1, \ldots, M), R_{i}>0$ and $C^{i}>0$ such that $\partial G \subset \cup \cup_{i=1}^{M} B_{i}$, where $B_{i}=B_{R_{i} / 4}\left(x_{i}\right)$, and (5.3) holds with $R_{0}=R_{i}$ and $C_{5}=C^{i}$. We note that $G_{1}:=G \backslash\left(\cup_{i=1}^{M} B_{i}\right)$ is compact and $G_{1} \subset G$. According to Lemma 4.16, for each $x_{0} \in G_{1}$, thete exist $R_{0}>0$ such that $B_{R_{0}}\left(x_{0}\right) \subset G$ and $C_{3}>0$ such that

$$
\|\nabla(\eta u)\|_{L^{p(\cdot)}(G)} \leq C_{3} \sup _{0 \neq v \in W_{0}^{1, p^{\prime}(\cdot)}\left(G \cap B_{R_{0}}\left(x_{0}\right)\right)} \frac{\left|\langle\nabla(\eta u), \nabla v\rangle_{G}\right|}{\|\nabla v\|_{L^{p^{\prime}(\cdot)}\left(G \cap B_{R_{0}}\left(x_{0}\right)\right)}}
$$

for all $u \in W_{0}^{1, p(\cdot)}(G)$ and $\left.\eta \in C_{0}^{\infty}\left(B_{R_{0} / 2}\right)\left(x_{0}\right)\right)$. Since $G_{1}$ is compact, there exist finitely many $x_{i} \in G$, $R_{i}>0$ and $C^{i}>0(i=M+1, \ldots, N)$ such that $G_{1} \subset \cup_{i=M+1}^{N} B_{i}$, where $B_{i}=B_{R_{i} / 4}\left(x_{i}\right)$ and (5.4) holds with $R_{0}=R_{i}$ and $C_{3}=C^{i}$.

For each $i=1, \ldots, N$, choose $\psi_{i} \in C_{0}^{\infty}\left(B_{i}^{\prime}\right)$, where $B_{i}^{\prime}=R_{R_{i} / 2}\left(x_{i}\right)$ such that $0 \leq \psi_{i} \leq 1, \psi_{i}=1$ on $B_{i}$ and denote $G_{i}=G \cap B_{R_{i} / 4}\left(x_{i}\right)$. Then from (5.3) and (5.4), we have

$$
\left\|\nabla u_{k}\right\|_{L^{p(\cdot)}\left(G_{i}\right)} \leq\left\|\nabla\left(\psi_{i} u_{k}\right)\right\|_{L^{p(\cdot)}(G)} \leq C^{i} \sup _{0 \neq v \in W_{0}^{1, p^{\prime}(\cdot)}\left(G_{i}\right)} \frac{\left|\left\langle\nabla\left(\psi_{i} u_{k}\right), \nabla v\right\rangle_{G}\right|}{\|\nabla v\|_{L^{p^{\prime}(\cdot)}\left(G_{i}\right)}}=: d_{k}^{i}
$$

Fix $i=1, \ldots, N$. For each $k \in \mathbb{N}$, there exists $v_{k} \in W_{0}^{1, p^{\prime}(\cdot)}\left(G_{i}\right)$ satisfying $\left\|\nabla v_{k}\right\|_{L^{p^{\prime}(\cdot)}\left(G_{i}\right)}=1$ and $0 \leq d_{k}^{i}-\left|\left\langle\nabla\left(\psi_{i} u_{k}\right), \nabla v_{k}\right\rangle_{G}\right| \leq 1 / k$. Therefore,

$$
\begin{aligned}
0 & \leq d_{k}^{i} \leq \frac{1}{k}+\left|\left\langle\nabla u_{k}, \nabla\left(\psi_{i} v_{k}\right)\right\rangle_{G}\right|+\left|\left\langle\nabla u_{k}, v_{k} \nabla \psi_{i}\right\rangle_{G}\right|+\left|\left\langle u_{k} \nabla \psi_{i}, \nabla v_{k}\right\rangle_{G}\right| \\
& \leq \frac{1}{k}+\varepsilon_{k}\left\|\nabla\left(\psi_{k} v_{k}\right)\right\|_{L^{p^{\prime}(\cdot)(G)}}+\left|\left\langle\nabla u_{k}, v_{k} \nabla \psi_{i}\right\rangle_{G}\right|+\left|\left\langle u_{k} \nabla \psi_{i}, \nabla v_{k}\right\rangle_{G}\right| .
\end{aligned}
$$

Using again the Poincaré inequality, we can see that the sequence $\left\{v_{k}\right\}_{k=1}^{\infty}$ is bounded in $W_{0}^{1, p^{\prime}(\cdot)}\left(G_{i}\right)$. Passing to a subsequence (still denoted by $\left\{v_{k}\right\}$ ), there exists $v \in W_{0}^{1, p^{\prime}(\cdot)}\left(G_{i}\right)$ such that $v_{k} \rightarrow v$ 
weakly in $W_{0}^{1, p^{\prime}(\cdot)}\left(G_{i}\right)$, so $v_{k} \rightarrow v$ strongly in $L^{p^{\prime}(\cdot)}\left(G_{i}\right)$. We estimate the right-hand side of (5.6). By the Hölder inequality,

$$
\begin{aligned}
\left|\left\langle\nabla u_{k}, v_{k} \nabla \psi_{i}\right\rangle_{G}\right| & \leq\left|\left\langle\nabla u_{k},\left(v_{k}-v\right) \nabla \psi_{i}\right\rangle_{G}\right|+\left|\left\langle\nabla u_{k}, v \nabla \psi_{i}\right\rangle_{G}\right| \\
& \leq 2\left\|\nabla u_{k}\right\|_{L^{p(\cdot)}(G)}\left\|\nabla \psi_{i}\right\|_{L^{\infty}\left(G_{i}\right)}\left\|v_{k}-v\right\|_{L^{p(\cdot)}\left(G_{i}\right)}+\left|\left\langle\nabla u_{k}, v \nabla \psi_{i}\right\rangle_{G}\right| \rightarrow 0 \text { as } k \rightarrow \infty
\end{aligned}
$$

and

$$
\left|\left\langle u_{k} \nabla \psi_{i}, \nabla v_{k}\right\rangle_{G}\right| \leq\left\|u_{k} \nabla \psi_{i}\right\|_{L^{p(\cdot)}\left(G_{i}\right)} \leq\left\|\nabla \psi_{i}\right\|_{L^{\infty}\left(B_{i}^{\prime}\right)}\left\|u_{k}\right\|_{L^{p(\cdot)}(G)} \rightarrow 0 \text { as } k \rightarrow \infty .
$$

By the Poincaré inequality,

$$
\left\|v_{k}\right\|_{L^{p^{\prime}(\cdot)\left(G_{i}\right)}} \leq c \operatorname{diam}\left(G_{i}\right)\left\|\nabla v_{k}\right\|_{L^{p^{\prime}(\cdot)}\left(G_{i}\right)}=c \operatorname{diam}\left(G_{i}\right) .
$$

Hence

$$
\left\|\nabla\left(\psi_{i} v_{k}\right)\right\|_{L^{p^{\prime}(\cdot)}(G)} \leq\left\|\left(\nabla \psi_{i}\right) v_{k}\right\|_{L^{p^{\prime}(\cdot)(G)}}+\left\|\psi_{i} \nabla v_{k}\right\|_{L^{p^{\prime}(\cdot)}\left(G_{i}\right)} \leq\left\|\nabla \psi_{i}\right\|_{L^{\infty}\left(B_{i}^{\prime}\right)} C \operatorname{diam}\left(G_{i}\right)+1
$$

Summing up the above, we see that $d_{k}^{i} \rightarrow 0$ as $k \rightarrow \infty$ for every $i=1, \ldots, N$. Since $G \subset \cup_{i=1}^{N} B_{i}$,

$$
\left\|\nabla u_{k}\right\|_{L^{p(\cdot)}(G)} \leq \sum_{i=1}^{N}\left\|\nabla u_{k}\right\|_{L^{p(\cdot)}\left(G_{i}\right)} \leq \sum_{i=1}^{N} d_{k}^{i} \rightarrow 0 \text { as } k \rightarrow \infty .
$$

This contradicts $\left\|\nabla u_{k}\right\|_{L^{p(\cdot)}(G)}=1$. This completes the proof of Theorem 3.1.

We can derive the $L^{p(\cdot)}$-regularity.

Theorem 5.2. Let $G$ be a bounded domain of $\mathbb{R}^{d}(d \geq 2)$ with a $C^{1}$-boundary. Assume that $p, q \in \mathcal{P}_{+}^{\log }(G)$ satisfies $q(x) \leq p(x)$ for all $x \in G$. If $u \in W_{0}^{1, q(\cdot)}(G)$ satisfies

$$
S_{p}(u):=\sup _{0 \neq \phi \in C_{0}^{\infty}(G)} \frac{\left|\langle\nabla u, \nabla \phi\rangle_{G}\right|}{\|\nabla \phi\|_{L^{p^{\prime}(\cdot)}(G)}}<\infty,
$$

then $u \in W_{0}^{1, p(\cdot)}(G)$ and

$$
\|\nabla u\|_{L^{p(\cdot)}(G)} \leq C_{p} S_{p}(u),
$$

where $C_{p}$ is the constant in Theorem 3.1.

Proof. Define a functional $F^{\prime}$ such that $F^{\prime}(\phi)=\langle\nabla u, \nabla \phi\rangle_{G}$ for $\phi \in \mathcal{D}(G)$. From (5.7),

$$
\left|F^{\prime}(\phi)\right| \leq\left\|F^{\prime}\right\|_{\left(W_{0}^{1, p^{\prime}(\cdot)}(G)\right)^{\prime}}\|\nabla \phi\|_{L^{p^{\prime}(\cdot)}(G)} \text { for } \phi \in \mathcal{D}(G),
$$

where $\left\|F^{\prime}\right\|_{\left(W_{0}^{1, p^{\prime}(\cdot)}(G)\right)^{\prime}}=S_{p}(u)$. Since $\mathcal{D}(G)$ is dense in $W_{0}^{1, p^{\prime}(\cdot)}(G)$ with respect to the norm $\| \nabla$.

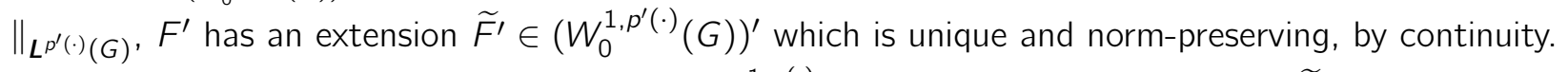
By Theorem 3.2, there exists uniquely $u_{p} \in W_{0}^{1, p(\cdot)}(G)$ such that $\left\langle\nabla u_{p}, \nabla \phi\right\rangle_{G}=\widetilde{F}^{\prime}(\phi)$ for all $\phi \in$ $W_{0}^{1, p^{\prime}(\cdot)}(G)$. Hence

$$
\left\langle\nabla u_{p}, \nabla \phi\right\rangle_{G}=\widetilde{F}^{\prime}(\phi)=F^{\prime}(\phi)=\langle\nabla u, \nabla \phi\rangle_{G}
$$


for all $\phi \in \mathcal{D}(G)$. Since $\mathcal{D}(G)$ is dense in $W_{0}^{1, q^{\prime}(\cdot)}(G)$ with respect to $\|\nabla \cdot\|_{L^{q^{\prime}(\cdot)}(G)^{- \text {norm }}}$ and $q(x) \leq$ $p(x)$ for all $x \in G$, so $u-u_{p} \in W_{0}^{1, q(\cdot)}(G)$, we have

$$
\left\langle\nabla\left(u-u_{p}\right), \nabla \phi\right\rangle_{G}=0 \text { for all } \phi \in W_{0}^{1, q^{\prime}(\cdot)}(G) .
$$

By Theorem 5.1, $u-u_{p}=0$, so $u=u_{p} \in W_{0}^{1, p(\cdot)}(G)$ and (3.1) holds, so (5.8) follows.

Corollary 5.3. Let $G$ be a bounded domain of $\mathbb{R}^{d}(d \geq 2)$ with a $C^{1}$-boundary. Assume that $p, q \in$ $\mathcal{P}_{+}^{\log }(G)$ satisfies $q(x) \leq p(x)$ for all $x \in G$. Suppose that $u \in W_{0}^{1, q(\cdot)}(G)$ and there exists $f \in L^{p(\cdot)}(G)$ such that

$$
\langle\nabla u, \nabla \phi\rangle_{G}=\langle\boldsymbol{f}, \boldsymbol{\nabla} \phi\rangle_{G} \text { for all } \phi \in \mathcal{D}(G) .
$$

Then $u \in W_{0}^{1, p(\cdot)}(G)$ and satisfies (5.7). Moreover, we have

$$
\|\nabla u\|_{L^{p(\cdot)}(G)} \leq 2 C_{p}\|\boldsymbol{f}\|_{L^{p(\cdot)}(G)},
$$

where $C_{p}$ is the constant in Theorem 3.1.

Proof. By the generalized Hölder inequality,

$$
\left|\langle\nabla u, \nabla \phi\rangle_{G}\right|=\left|\langle\boldsymbol{f}, \nabla \phi\rangle_{G}\right| \leq 2\|\boldsymbol{f}\|_{L^{p(\cdot)}(G)}\|\nabla \phi\|_{L^{p^{\prime}(\cdot)}(G)} \text { for all } \phi \in \mathcal{D}(G) .
$$

Hence (5.7) holds and $S_{p}(u) \leq 2\|\boldsymbol{f}\|_{L^{p(\cdot)}(G)}$. Hence we have

$$
\|\nabla u\|_{L^{p(\cdot)}(G)} \leq C_{p} S_{p}(u) \leq 2 C_{p}\|\boldsymbol{f}\|_{L^{p(\cdot)}(G)} .
$$

\section{Dirichlet problem for the Poisson equation}

Let $G$ be a bounded domain of $\mathbb{R}^{d}(d \geq 2)$ with a $C^{1}$-boundary $\partial G$. We consider the following Dirichlet problem for the Poisson equation.

$$
\begin{cases}-\Delta u=f & \text { in } G, \\ u=g & \text { on } \partial G .\end{cases}
$$

We are in a position to state the main theorem of this section.

Theorem 6.1. Let $G$ be a bounded domain of $\mathbb{R}^{d}(d \geq 2)$ with a $C^{1}$-boundary $\partial G$ and let $p \in \mathcal{P}_{+}^{\log }(G)$. Assume that $f \in W^{-1, p(\cdot)}(G)$ and $g \in \operatorname{Tr}\left(W^{1, p(\cdot)}(G)\right)$. Then the system (6.1) has a unique weak solution $u \in W^{1, p(\cdot)}(G)$ in the sense that $\left.u\right|_{\partial G}=g$ and

$$
\langle\nabla u, \nabla v\rangle_{G}=\langle f, v\rangle_{W^{-1, p(\cdot)}(G), W_{0}^{1, p^{\prime}(\cdot)}(G)} \text { for all } v \in W_{0}^{1, p^{\prime}(\cdot)}(G) .
$$

Furthermore, there exists a constant $C=C(p, d, G)>0$ such that

$$
\|u\|_{W^{1, p(\cdot)}(G)} \leq C\left(\|f\|_{W^{-1, p(\cdot)}(G)}+\|g\|_{\operatorname{Tr}\left(W^{1, p(\cdot)}(G)\right)}\right) .
$$


Proof. First we reduce the problem (6.1) to the homogeneous Dirichlet problem. Since $g \in$ $\operatorname{Tr}\left(W^{1, p(\cdot)}(G)\right)$, there exists $w \in W^{1, p(\cdot)}(G)$ such that $\left.w\right|_{\partial G}=g$ and

$$
\|W\|_{W^{1, p(\cdot)}(G)}=\|g\|_{\operatorname{Tr}\left(W^{1, p(\cdot)}(G)\right)} .
$$

Indeed, by definition of $\|g\|_{\operatorname{Tr}\left(W^{1, p(\cdot)}(G)\right)}$, there exists $\left\{w_{j}\right\} \subset W^{1, p(\cdot)}(G)$ with $\left.w_{j}\right|_{\partial G}=g$ and

$$
\|g\|_{\operatorname{Tr}\left(W^{1, p(\cdot)}(G)\right)}=\lim _{j \rightarrow \infty}\left\|W_{j}\right\|_{\left.W^{1, p(\cdot)}(G)\right)} .
$$

Hence $\left\{w_{j}\right\}$ is bounded in a reflexive Banach space $W^{1, p(\cdot)}(G)$, so passing to a subsequence of $\left\{w_{j}\right\}$ (still denoted by $\left\{w_{j}\right\}$ ) we may assume that $w_{j} \rightarrow W$ weakly in $W^{1, p(\cdot)}(G)$. By Lemma 2.6, $\left.w_{j}\right|_{\partial G} \rightarrow$ $\left.w\right|_{\partial G}$ in $L^{p(\cdot)}(\partial G)$, so $\left.w\right|_{\partial G}=g$. Therefore,

$$
\|g\|_{\operatorname{Tr}\left(W^{1, p(\cdot)}(G)\right)} \leq\|W\|_{W^{1, p(\cdot)}(G)} \leq \liminf _{j \rightarrow \infty}\left\|W_{j}\right\|_{W^{1, p(\cdot)}(G)}=\|g\|_{\operatorname{Tr}\left(W^{1, p(\cdot)}(G)\right)} .
$$

Since $\Delta w \in W^{-1, p(\cdot)}(G)$, if we replace an unknown function $u$ with $v=u-w$ and a known function $f$ with $F=f+\Delta w \in W^{-1, p(\cdot)}(G)$, the problem (6.1) is reduced the following problem.

$$
\begin{cases}-\Delta v=F & \text { in } G \\ v=0 & \text { on } \partial G\end{cases}
$$

Therefore, we consider (6.5), that is, find $v \in W_{0}^{1, p(\cdot)}(G)$ such that

$$
\langle\nabla v, \nabla \phi\rangle_{G}=\langle F, \phi\rangle_{W^{-1, p(\cdot)}(G), W_{0}^{1, p^{\prime}(\cdot)}(G)} \text { for all } \phi \in W_{0}^{1, p^{\prime}(\cdot)}(G) .
$$

According to Theorem 3.2, there exists a unique $v \in W_{0}^{1, p(\cdot)}(G)$ such that (6.6) holds and

$$
\begin{aligned}
C_{p}^{-1}\|\nabla v\|_{L^{p(\cdot)}(G)} & \leq\|F\|_{W^{-1, p(\cdot)}(G)} \\
& \leq\|f\|_{W^{-1, p(\cdot)}(G)}+\|\Delta W\|_{W^{-1, p(\cdot)}(G)} \\
& \leq\|f\|_{W^{-1, p(\cdot)}(G)}+C(p, G)\|W\|_{W^{1, p(\cdot)}(G)} \\
& \leq\|f\|_{W^{-1, p(\cdot)}(G)}+C(p, G)\|g\|_{\operatorname{Tr}\left(W^{1, p(\cdot)}(G)\right)} .
\end{aligned}
$$

By the Poincaré inequality, $\|v\|_{W^{1, p(\cdot)}(G)} \leq C_{1}(p, G)\|\nabla v\|_{L^{p(\cdot)}(G)}$. If we put $u=v+w,(6.2)$ and the estimate (6.3) follows.

Remark 6.2. The authors in [11] showed that if $G$ is a bounded domain with a $C^{1,1}$-boundary and $f \in L^{p(\cdot)}(G), g \in \operatorname{Tr}\left(W^{2, p(\cdot)}(G)\right)$, the system (6.1) has a unique strong solution $u \in W^{2, p(\cdot)}(G)$ and there exists a constant $C$ depending only on $p$ and $G$ such that

$$
\|u\|_{W^{2, p(\cdot)}(G)} \leq C\left(\|f\|_{L^{p(\cdot)}(G)}+\|g\|_{\operatorname{Tr}\left(W^{2, p(\cdot)}(G)\right)}\right) .
$$

They used the Newton potential, and only announced the existence of a weak solution as in Theorem 6.1. However, we can easily show that Theorem 6.1 holds using Theorem 3.1 and 3.2 under the weaker assumption of the regularity of boundary. 
7. An approach to the Stokes problem

In this section, let $G$ be a bounded domain of $\mathbb{R}^{d}(d \geq 2)$ with a $C^{1}$-boundary $\Gamma=\partial G$. We consider the following homogeneous Stokes problem.

$$
\begin{cases}-\Delta \boldsymbol{u}+\nabla \pi=\boldsymbol{f} & \text { in } G \\ \operatorname{div} \boldsymbol{u}=0 & \text { in } \Omega \\ \boldsymbol{u}=0 & \text { on } \Gamma, \\ \pi=0 & \text { on } \Gamma .\end{cases}
$$

We have the following theorem.

Theorem 7.1. Let $G$ be a bounded domain of $\mathbb{R}^{d}(d \geq 2)$ with a $C^{1}$-boundary $\Gamma=\partial G$ and let $p \in \mathcal{P}_{+}^{\log }(G)$. Assume that $f \in L^{p(\cdot)}(G)$. Then the problem (7.1) has a unique weak solution $(\boldsymbol{u}, \pi) \in \boldsymbol{W}_{0}^{1, p(\cdot)}(G) \times W_{0}^{1, p(\cdot)}(G)$, in the sense of

$$
\langle\nabla u, \nabla v\rangle_{G}+\langle\nabla \pi, v\rangle_{G}=\langle\boldsymbol{f}, \boldsymbol{v}\rangle_{G} \text { for all } \boldsymbol{v} \in \boldsymbol{W}_{0}^{1, p^{\prime}(\cdot)}(G),
$$

and there exists a constant $C=C(p, d, G)>0$ such that

$$
\|\boldsymbol{u}\|_{W^{1, p(\cdot)}(G)}+\|\pi\|_{W^{1, p(\cdot)}(G)} \leq C\|\boldsymbol{f}\|_{L^{p(\cdot)}(G)} .
$$

Furthermore, if $G$ is of class $C^{1,1}$, then $\boldsymbol{u} \in \boldsymbol{W}^{2, p(\cdot)}(G)$ and $(\boldsymbol{u}, \pi)$ is a strong solution of (7.1). Moreover, we have

$$
\|\boldsymbol{u}\|_{W^{2, p(\cdot)}(G)}+\|\pi\|_{W^{1, p(\cdot)}(G)} \leq C^{\prime}\|\boldsymbol{f}\|_{L^{p(\cdot)}(G)}
$$

where $C^{\prime}$ is a constant depending only on $p, d$ and $G$.

Proof. First we consider the following Dirichlet problem for the Laplacian $\Delta$.

$$
\begin{cases}\Delta \pi=\operatorname{div} f & \text { in } G, \\ \pi=0 & \text { on } \Gamma .\end{cases}
$$

Suppose that $f \in L^{p(\cdot)}(G)$. Since $\operatorname{div} f \in W^{-1, p(\cdot)}(G)$, if follows from Theorem 6.1 that (7.4) has a unique weak solution $\pi \in W_{0}^{1, p(\cdot)}(G)$ and there exist positive constants $C=C(p, d, G)$ and $C_{1}=C_{1}(p, d, G)$ such that

$$
\|\pi\|_{W^{1, p(\cdot)}(G)} \leq C\|\operatorname{div} \boldsymbol{f}\|_{W^{-1, p(\cdot)}(G)} \leq C_{1}\|\boldsymbol{f}\|_{L^{p(\cdot)}(G)}
$$

We note that

$$
f-\nabla \pi \in L^{p(\cdot)}(G) \text { and } \operatorname{div}(f-\nabla \pi)=0 \text { in the distribution sense. }
$$

We apply Proposition 3.3. For this purpose, define

$$
\boldsymbol{X}^{p(\cdot)}(G)=\left\{\boldsymbol{u} \in \boldsymbol{W}_{0}^{1, p(\cdot)}(G) ; \operatorname{div} \boldsymbol{u}=0 \text { in } G\right\}
$$


Then clearly $\boldsymbol{X}^{p(\cdot)}(G)$ is a closed subspace of a reflexive Banach space $\boldsymbol{W}_{0}^{1, p(\cdot)}(G)$, so $\boldsymbol{X}^{p(\cdot)}(G)$ is also reflexive Banach space. Since $\boldsymbol{X}^{p(\cdot)}(G) \subset \boldsymbol{W}_{0}^{1, p(\cdot)}(G)$, if follows from Theorem 3.1

$$
\begin{aligned}
0<C_{p}^{-1} & \leq \inf _{\boldsymbol{0} \neq \boldsymbol{u} \in \boldsymbol{W}_{0}^{1, p(\cdot)}(G)} \sup _{\boldsymbol{0} \neq \boldsymbol{v} \in \boldsymbol{W}_{0}^{1, p^{\prime}(\cdot)}(G)} \frac{\left|\langle\boldsymbol{\nabla} \boldsymbol{v}, \boldsymbol{\nabla} \boldsymbol{u}\rangle_{G}\right|}{\|\boldsymbol{\nabla} \boldsymbol{u}\|_{\boldsymbol{L}^{p(\cdot)}(G)}|| \boldsymbol{\nabla} \boldsymbol{v} \|_{\boldsymbol{L}^{p^{\prime}(\cdot)}(G)}} \\
& \leq \inf _{\boldsymbol{0} \neq \boldsymbol{u} \in \boldsymbol{X}^{p(\cdot)(G)}} \sup _{\boldsymbol{0} \neq \boldsymbol{v} \in \boldsymbol{W}_{0}^{1, p^{\prime}(\cdot)}(G)} \frac{\left|\langle\boldsymbol{\nabla} \boldsymbol{v}, \boldsymbol{\nabla} \boldsymbol{u}\rangle_{G}\right|}{\|\boldsymbol{\nabla} \boldsymbol{u}\|_{\boldsymbol{L}^{p(\cdot)}(G)}\|\boldsymbol{\nabla} \boldsymbol{v}\|_{\boldsymbol{L}^{p^{\prime}(\cdot)}(G)}} .
\end{aligned}
$$

Taking the Poincaré inequality into consideration, let $X=W_{0}^{1, p^{\prime}(\cdot)}(G)$ equipped with the norm $\|\boldsymbol{v}\|_{X}=$ $\|\boldsymbol{\nabla} \boldsymbol{v}\|_{L^{p^{\prime}(\cdot)}(G)}$ and $M=\boldsymbol{X}^{p(\cdot)}(G)$ equipped with the norm $\|\boldsymbol{u}\|_{M}=\|\boldsymbol{\nabla} \boldsymbol{u}\|_{L^{p(\cdot)}(G)}$. Define $a(\boldsymbol{v}, \boldsymbol{u})=$ $\langle\nabla \boldsymbol{v}, \boldsymbol{\nabla} \boldsymbol{u}\rangle_{G}$ for $\boldsymbol{v} \in X, \boldsymbol{u} \in M$. By the generalized Hölder inequality, we have

$$
|a(\boldsymbol{v}, \boldsymbol{u})| \leq 2\|\boldsymbol{\nabla} \boldsymbol{v}\|_{L^{p^{\prime}(\cdot)(G)}}\|\boldsymbol{\nabla} \boldsymbol{u}\|_{L^{p(\cdot)}(G)}=2\|\boldsymbol{v}\|_{X}\|\boldsymbol{u}\|_{M} .
$$

Thus $a(\boldsymbol{v}, \boldsymbol{u})$ is a continuous bilinear form on $X \times M$. Define bounded linear operators $A: X \rightarrow M^{\prime}$ and $A^{\prime}: M \rightarrow X^{\prime}$ by $\langle A \boldsymbol{v}, \boldsymbol{u}\rangle=\left\langle\boldsymbol{v}, A^{\prime} \boldsymbol{u}\right\rangle=a(\boldsymbol{v}, \boldsymbol{u})$. Then $\left|\left\langle\boldsymbol{v}, A^{\prime} \boldsymbol{u}\right\rangle\right| \leq 2\|\boldsymbol{v}\|_{X}\|\boldsymbol{u}\|_{M}$ for all $\boldsymbol{v} \in X, \boldsymbol{u} \in M$. From (7.7),

Put $V=\operatorname{Ker} A$.

$$
C_{p}^{-1} \leq \inf _{\boldsymbol{0} \neq \boldsymbol{u} \in M} \sup _{\mathbf{0} \neq \boldsymbol{v} \in X} \frac{|a(\boldsymbol{v}, \boldsymbol{u})|}{\|\boldsymbol{v}\|_{X}\|\boldsymbol{u}\|_{M}}
$$

We characterize $V=\operatorname{Ker} A$.

Lemma 7.2. It follows that $V=\left\{\boldsymbol{v}=(-\Delta)^{-1} \nabla \varphi ; \varphi \in L^{p^{\prime}(\cdot)}(G)\right\}$. Here $\boldsymbol{v}=(-\Delta)^{-1} \boldsymbol{g}, \boldsymbol{g} \in$ $W^{-1, p^{\prime}(\cdot)}(G)$ means that $\boldsymbol{v} \in W_{0}^{1, p^{\prime}(\cdot)}(G)$ is a unique weak solution of the following problem.

$$
\begin{cases}-\Delta \boldsymbol{v}=\boldsymbol{g} & \text { in } G \\ \boldsymbol{v}=0 & \text { on } \Gamma .\end{cases}
$$

Proof. Let $\boldsymbol{v} \in V$. Then $\langle A \boldsymbol{v}, \boldsymbol{u}\rangle_{M^{\prime}, M}=0$ for all $\boldsymbol{u} \in M$, that is,

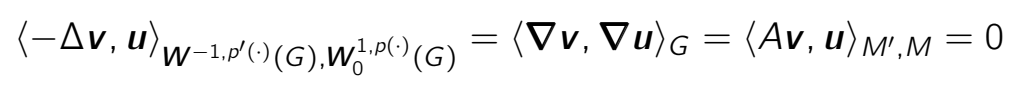

for all $\boldsymbol{u} \in \boldsymbol{X}^{p(\cdot)}(G)$. By the de Rham theorem (cf. Aramaki $[3,4]$ ), there exists $\varphi \in L^{p^{\prime}(\cdot)}(G)$ such that $-\Delta \boldsymbol{v}=\nabla \varphi$ in $\boldsymbol{W}^{-1, p^{\prime}(\cdot)}(G)$. Since $\boldsymbol{v}=0$ on $\Gamma$, we have $\boldsymbol{v}=(-\Delta)^{-1} \nabla \varphi$. Thus we have

$$
V \subset\left\{\boldsymbol{v}=(-\Delta)^{-1} \nabla \varphi ; \varphi \in L^{p^{\prime}(\cdot)}(G)\right\} .
$$

Conversely, let $\boldsymbol{v}=(-\Delta)^{-1} \nabla \varphi$ for some $\varphi \in L^{p^{\prime}(\cdot)}(G)$. Then $\boldsymbol{v}$ is a unique weak solution of (7.8) with $\boldsymbol{g}=\boldsymbol{\nabla} \varphi$. For any $\boldsymbol{u} \in \boldsymbol{X}^{p(\cdot)}(G)$, we have

$$
\begin{aligned}
\langle A \boldsymbol{v}, \boldsymbol{u}\rangle_{M^{\prime}, M}=\langle\boldsymbol{\nabla} \boldsymbol{v}, \boldsymbol{\nabla} \boldsymbol{u}\rangle_{G}=\langle-\Delta \boldsymbol{v}, \boldsymbol{u}\rangle_{\boldsymbol{W}^{-1, p^{\prime}(\cdot)}(G), \boldsymbol{W}_{0}^{1, p(\cdot)}(G)} & \\
& =\langle\boldsymbol{\nabla} \varphi, \boldsymbol{u}\rangle_{\boldsymbol{W}^{-1, p^{\prime}(\cdot)}(G), W_{0}^{1, p(\cdot)}(G)}=-\langle\varphi, \operatorname{div} \boldsymbol{u}\rangle_{G}=0 .
\end{aligned}
$$

Hence $A \boldsymbol{v}=0$ in $M^{\prime}$, that is, $\boldsymbol{v} \in \operatorname{Ker} A=V$.

Denote that $V^{\perp}=\left\{\boldsymbol{f} \in X^{\prime}=\boldsymbol{W}^{-1, p(\cdot)}(G) ;\langle\boldsymbol{f}, \boldsymbol{v}\rangle_{X^{\prime}, X}=0\right.$ for all $\left.\boldsymbol{v} \in V\right\}$. 
Lemma 7.3. If $\boldsymbol{g} \in \boldsymbol{L}^{p(\cdot)}(G)$ satisfies $\operatorname{div} \boldsymbol{g}=0$, then $\boldsymbol{g} \in V^{\perp}$.

Proof. According to Lemma 7.2, for any $\boldsymbol{v} \in V$, there exists $\varphi \in L^{p^{\prime}(\cdot)}(G)$ such that $\boldsymbol{v}=(-\Delta)^{-1} \nabla \varphi$. Thereby, we have

$$
\begin{aligned}
\langle\boldsymbol{g}, \boldsymbol{v}\rangle_{X^{\prime}, X} & =\left\langle\boldsymbol{g},(-\Delta)^{-1} \boldsymbol{\nabla} \varphi\right\rangle_{W^{-1, p(\cdot)}(G), W_{0}^{1, p^{\prime}(\cdot)}(G)} \\
& =\left\langle(-\Delta)^{-1} \boldsymbol{g}, \boldsymbol{\nabla} \varphi\right\rangle_{\boldsymbol{W}_{0}^{1, p(\cdot)}(G), W^{-1, p^{\prime}(\cdot)}(G)} \\
& =-\left\langle\operatorname{div}(-\Delta)^{-1} \boldsymbol{g}, \varphi\right\rangle_{G} .
\end{aligned}
$$

If we put $\boldsymbol{w}=(-\Delta)^{-1} \boldsymbol{g}$, we have $-\Delta \boldsymbol{w}=\boldsymbol{g}$ in $\Omega$ and $\boldsymbol{w}=0$ on $\Gamma$. Hence

$$
(-\Delta) \operatorname{div} \boldsymbol{w}=-\operatorname{div} \Delta \boldsymbol{w}=\operatorname{div} \boldsymbol{g}=0 .
$$

Therefore, $(-\Delta)^{-1}(-\Delta) \operatorname{div} \boldsymbol{w}=\operatorname{div} \boldsymbol{w}=0$, that is, $\operatorname{div}(-\Delta)^{-1} \boldsymbol{g}=0$. This implies that $\langle\boldsymbol{g}, \boldsymbol{v}\rangle_{X^{\prime}, X}=0$ for all $v \in V$. Thus $g \in V^{\perp}$.

We continue the proof of Theorem 7.1. From (7.6), we know that $f-\nabla \pi \in L^{p(\cdot)}(G)$ and $\operatorname{div}(\boldsymbol{f}-\boldsymbol{\nabla} \pi)=0$. By Proposition 3.3, $A^{\prime}: M \rightarrow V^{\perp}$ is an isomorphism and $C_{p}$ is the continuity constant of $\left(A^{\prime}\right)^{-1}$. By Lemma 7.3, we see that $f-\nabla \pi \in V^{\perp}$, so there exists a unique $\boldsymbol{u} \in M$ such that $A^{\prime} \boldsymbol{u}=\boldsymbol{f}-\boldsymbol{\nabla} \pi$, that is, $\operatorname{div} \boldsymbol{u}=0$ in $G, \boldsymbol{u}=0$ on $\Gamma$ and

$$
\langle\nabla v, \nabla u\rangle_{G}=\langle\boldsymbol{v}, \boldsymbol{f}-\nabla \pi\rangle_{X, X^{\prime}} \text { for all } \boldsymbol{v} \in \boldsymbol{W}_{0}^{1, p^{\prime}(\cdot)}(G),
$$

so (7.2) holds. Furthermore, we have

$$
\begin{aligned}
\|\boldsymbol{u}\|_{W^{1, p(\cdot)}(G)} & \leq C_{p}\|\boldsymbol{f}-\nabla \pi\|_{W^{-1, p(\cdot)}(G)} \\
& \leq C_{p}^{\prime}\|\boldsymbol{f}-\nabla \pi\|_{L^{p(\cdot)}(G)} \\
& \leq C_{p}^{\prime}\left(\|\boldsymbol{f}\|_{L^{p(\cdot)}(G)}+\|\nabla \pi\|_{L^{p(\cdot)}(G)}\right) \\
& \leq C_{p}^{\prime \prime}\|\boldsymbol{f}\|_{L^{p(\cdot)}(G)} .
\end{aligned}
$$

Summing up this inequality and (7.5), we get the estimate (7.3).

If, in particular, $G$ is of class $C^{1,1}$, since $-\Delta \boldsymbol{u}=\boldsymbol{f}-\boldsymbol{\nabla} \pi \in \boldsymbol{L}^{p(\cdot)}(G)$ in $G$ and $\boldsymbol{u}=\mathbf{0}$ on $\Gamma$, it follows from [11, Theorem 14.1.2] that $\boldsymbol{u} \in \boldsymbol{W}^{2, p(\cdot)}(G)$ and

$$
\|\boldsymbol{u}\|_{\boldsymbol{W}^{2, p(\cdot)}(G)} \leq C\|\boldsymbol{f}-\nabla \pi\|_{L^{p(\cdot)}(G)} \leq C_{1}\|\boldsymbol{f}\|_{L^{p(\cdot)}(G)}
$$

Now we consider the inhomogeneous Stokes problem.

$$
\begin{cases}-\Delta \boldsymbol{u}+\nabla \pi=\boldsymbol{f} & \text { in } G \\ \operatorname{div} \boldsymbol{u}=\varphi & \text { in } G \\ \boldsymbol{u}=\boldsymbol{g} & \text { on } \Gamma, \\ \pi=\pi_{0} & \text { on } \Gamma .\end{cases}
$$


Theorem 7.4. Let $G$ be a bounded domain of $\mathbb{R}^{d}$ with a $C^{1,1}$-boundary $\Gamma$ and let $p \in \mathcal{P}_{+}^{\log }(G)$. Assume that $\boldsymbol{f} \in \boldsymbol{L}^{p(\cdot)}(G), \pi_{0} \in \operatorname{Tr}\left(W^{1, p(\cdot)}(G)\right), \varphi \in W^{1, p(\cdot)}(G)$ and $\boldsymbol{g} \in \operatorname{Tr}\left(\boldsymbol{W}^{2, p(\cdot)}(G)\right)$ satisfy the compatibility condition

$$
\int_{G} \varphi d x=\int_{\Gamma} \boldsymbol{g} \cdot \boldsymbol{n} d \sigma
$$

where $d \sigma$ is the surface measure on $\Gamma$. Then there exists a unique solution $(\boldsymbol{u}, \pi) \in \boldsymbol{W}^{2, p(\cdot)}(G) \times$ $W^{1, p(\cdot)}(G)$ of $(7.9)$ and there exists a constant $C=C(p, d, G)>0$ such that

$$
\begin{aligned}
&\|\boldsymbol{u}\|_{\boldsymbol{W}^{2, p(\cdot)}(G)}+\|\pi\|_{W^{1, p(\cdot)}(G)} \\
& \leq C\left(\|\boldsymbol{f}\|_{L^{p(\cdot)}(G)}+\left\|\pi_{0}\right\|_{\operatorname{Tr}\left(W^{1, p(\cdot)}(G)\right)}+\|\varphi\|_{W^{1, p(\cdot)}(G)}+\|\boldsymbol{g}\|_{\operatorname{Tr}\left(\boldsymbol{W}^{1, p(\cdot)}(G)\right)}\right) .
\end{aligned}
$$

Before the proof, it is necessary to prepare some arguments.

Proposition 7.5. Let $G$ be a bounded domain of $\mathbb{R}^{d}$ with a $C^{1,1}$-boundary $\Gamma$ and $p \in \mathcal{P}_{+}^{\log }(G)$. If we assume that $g \in \operatorname{Tr}\left(W^{1, p(\cdot)}(G)\right)$, then there exists $u \in W^{2, p(\cdot)}(\Omega)$ such that $\gamma_{1}(u)=g$ and $\gamma_{0}(u)=0$.

Proof. We use the argument of Boyer and Fabrie [7, Proof of Theorem III.2.23].

Let $\delta(x)$ be the signed distance from $x$ to $\Gamma$, that is,

$$
\delta(x)= \begin{cases}d(x, \Gamma) & \text { if } x \in \bar{G} \\ -d(x, \Gamma) & \text { if } x \notin G .\end{cases}
$$

Then $\delta$ is Lipschitz-continuous in $\mathbb{R}^{d}$ with the Lipschitz constant $\operatorname{Lip}(\delta) \leq 1$. Let $\eta$ be a standard mollifier, that is, $\eta \in C_{0}^{\infty}\left(\mathbb{R}^{d}\right)$, supp $\eta \subset B$ (the unit sphere of $\mathbb{R}^{d}$ ), $\eta \geq 0, \int_{\mathbb{R}^{d}} \eta d x=\int_{B} \eta d x=1$ and $\eta(x)$ only depends on $|x|$.

For $x \in \mathbb{R}^{d}$ and $\tau \in \mathbb{R}$, define a function

$$
G(x, \tau)=\int_{B} \delta\left(x+\frac{\tau}{2} z\right) \eta(z) d x
$$

Then we can clearly see that $G \in C^{\infty}\left(\mathbb{R}^{d} \times(\mathbb{R} \backslash\{0\})\right.$ and

$$
\left|G\left(x, \tau_{1}\right)-G\left(x, \tau_{2}\right)\right| \leq \frac{1}{2}\left|\tau_{1}-\tau_{2}\right| .
$$

Therefore, by Banach fixed-point theorem, for any $x \in \mathbb{R}^{d}$, there exists uniquely $\rho(x) \in \mathbb{R}$ such that $\rho(x)=G(x, \rho(x))$. We call $\rho$ a regularized distance function of $G$. The regularized distance function $\rho$ has the following properties.

(i) $\rho(x)=0 \Longleftrightarrow \delta(x)=0 \Longleftrightarrow x \in \Gamma$, and there exists constants $C_{1}, C_{2}>0$ such that $C_{1} \leq$ $\delta(x) / \rho(x) \leq C_{2}$ for $x \in \mathbb{R}^{d} \backslash \Gamma$.

(ii) $\rho \in C^{1,1}\left(\mathbb{R}^{d}\right) \cap C^{\infty}\left(\mathbb{R}^{d} \backslash \Gamma\right)$.

(iii) $\boldsymbol{\nabla} \rho(x)=\boldsymbol{\nabla} \delta(x)=-\boldsymbol{n}(x)$ for all $x \in \Gamma$, and there exists an open neighborhood $U$ of $\Gamma$ such that $\inf _{U}|\nabla \rho|>0$. 
For $g \in \operatorname{Tr}\left(W^{1, p(\cdot)}(G)\right)$, there exists $v \in W^{1, p(\cdot)}(G)$ such that $\gamma_{0}(v)=g$ and there exists a constant $C>0$ such that $\|v\|_{W^{1, p(\cdot)}(G)} \leq C\|g\|_{\operatorname{Tr}\left(W^{1, p(\cdot)}(G)\right)}$. We define

$$
R_{n} g(x)=-\rho(x) \int_{B} v(x+\alpha \rho(x) z) \eta(z) d z
$$

where $\alpha>0$ so that $\alpha \leq C_{1}$ and $\alpha \operatorname{Lip}(\rho)<1, C_{1}$ is the constant of (i). For $x \in G$ and $z \in B$, we have $x+\alpha \rho(x) z \in G$, so $R_{n} g$ is well defined and $R_{n} g \in C^{\infty}(G)$. We show that $R_{n} g \in W^{2, p(\cdot)}(G)$. By the calculations, for $i, j=1, \ldots, d$, we have

$$
\begin{aligned}
\frac{\partial R_{\boldsymbol{n}} g}{\partial x_{i}}(x)=- & \frac{\partial \rho}{\partial x_{i}}(x) \int_{B} v(x+\alpha \rho(x) z) \eta(z) d z \\
- & \rho(x) \int_{B} \nabla v(x+\alpha \rho(x) z) \cdot\left(e_{i}+\alpha \frac{\partial \rho}{\partial x_{i}}(x) z\right) \eta(z) d z, \\
\frac{\partial^{2} R_{\boldsymbol{n}} g}{\partial x_{i} \partial x_{j}}= & -\frac{\partial^{2} \rho}{\partial x_{i} \partial x_{j}}(x) \int_{B} v(x+\alpha \rho(x) z) \psi(z) d z \\
& -\frac{\partial \rho}{\partial x_{i}}(x) \int_{B} \frac{\partial v}{\partial x_{j}}(x+\alpha \rho(x) z) \psi(z) d z \\
& -\alpha \frac{\partial \rho}{\partial x_{i}}(x) \frac{\partial \rho}{\partial x_{j}}(x) \int_{B} \nabla v(x+\alpha \rho(x) z) \cdot \psi(z) z d z \\
& +(d-1) \frac{\partial \rho}{\partial x_{j}}(x) \int_{B} \frac{\partial v}{\partial x_{i}}(x+\alpha \rho(x) z) \eta(z) d z \\
& +\frac{1}{\alpha} \int_{B} \frac{\partial v}{\partial x_{i}}(x+\alpha \rho(x) z) \frac{\partial \eta}{\partial z_{j}}(z) d z \\
& +\frac{\partial \rho}{\partial x_{j}}(x) \int_{B} \frac{\partial v}{\partial x_{i}}(x+\alpha \rho(x) z) \nabla \eta(z) \cdot z d z
\end{aligned}
$$

where $\psi(z)=\eta(z)-\operatorname{div}_{z}(\eta(z) z)$. Since all the derivatives of $\rho$ up to second-order are bounded, it suffices to show that the terms of the form

$$
F(x)=\int_{B} f(x+\alpha \rho(x) z) \widetilde{\psi}(z) d z \text { for } x \in G
$$

where $f \in L^{p(\cdot)}(G)$ and $\widetilde{\psi} \in C_{0}^{\infty}(B)$, belong to $L^{p(\cdot)}(G)$.

We note that we can not use the Jensen inequality in the case of variable exponent. However, applying a variant of the Jensen inequality (cf. [11, Theorem 4.2.4 and Corollary 4.2.5]), there exists a constant $C>0$ such that

$$
\rho_{p(\cdot), G}(F) \leq C\|f\|_{L^{p(\cdot)}(G)}^{p^{+}-p^{-}} \rho_{p(\cdot), G}(f)+C\|f\|_{L^{p(\cdot)}(G)}^{p^{+}} .
$$

Therefore, we see that $F \in L^{p(\cdot)}(G)$, so $u:=R_{n} g \in W^{2, p(\cdot)}(G)$. From (7.11) and property (i), we see that $\gamma_{0}(u)=0$. From $(7.12)$ and property (iii), we can see that $\gamma_{1}(u)=\gamma_{0}\left(\nabla R_{\boldsymbol{n}} g\right) \cdot \boldsymbol{n}=\gamma_{0}(v)=$ g. 
Lemma 7.6. Let $G$ be a bounded domain of $\mathbb{R}^{d}$ with a $C^{1,1}$-boundary $\Gamma$ and let $p \in \mathcal{P}_{+}^{\log }(G)$. For $\left(g_{0}, g_{1}\right) \in \operatorname{Tr}\left(W^{2, p(\cdot)}(G)\right) \times \operatorname{Tr}\left(W^{1, p(\cdot)}(G)\right)$, there exists $u \in W^{2, p(\cdot)}(G)$ such that $\gamma_{0}(u)=g_{0}$ and $\gamma_{1}(u)=g_{1}$, moreover there exists a constant $C>0$ such that

$$
\|u\|_{W^{2, p(\cdot)}(G)} \leq C\left(\left\|g_{0}\right\|_{\operatorname{Tr}\left(W^{2, p(\cdot)}(G)\right)}+\left\|g_{1}\right\|_{\operatorname{Tr}\left(W^{1, p(\cdot)}(G)\right)}\right) .
$$

Proof. From Theorem 2.5, we see that

$$
W_{0}^{2, p(\cdot)}(G)=\left\{v \in W^{2, p(\cdot)}(G) ; \gamma_{0}(v)=\gamma_{1}(v)=0\right\}
$$

We consider the mapping

$$
\gamma: W^{2, p(\cdot)}(G) / W_{0}^{2, p(\cdot)}(G) \ni[u] \mapsto\left(\gamma_{0}(u), \gamma_{1}(u)\right) \in \operatorname{Tr}\left(W^{2, p(\cdot)}(G)\right) \times \operatorname{Tr}\left(W^{1, p(\cdot)}(G)\right) .
$$

Since $\boldsymbol{n}$ is a Lipschitz function on $\Gamma$, we can extend $\boldsymbol{n}$ to a Lipschitz function on $\bar{G}$, so $\gamma_{1}(u)=$ $\gamma_{0}(\nabla u \cdot \boldsymbol{n})$. Thus $\gamma$ is a linear continuous injection. We show that $\gamma$ is surjective. Let $\left(g_{0}, g_{1}\right) \in$ $\operatorname{Tr}\left(W^{2, p(\cdot)}(G)\right) \times \operatorname{Tr}\left(W^{1, p(\cdot)}(G)\right)$. Choose $v_{0} \in W^{2, p(\cdot)}(G)$ such that $\gamma_{0}\left(v_{0}\right)=g_{0}$ and define $v=$ $v_{0}+R_{n}\left(g_{1}-\gamma_{1}\left(v_{0}\right)\right) \in W^{2, p(\cdot)}(G)$. Then by Proposition 7.5, $\gamma_{0}(v)=\gamma_{0}\left(v_{0}\right)=g_{0}$ and $\gamma_{1}(v)=g_{1}$. Thereby $\gamma$ is surjective. By the Banach open mapping theorem, $\gamma^{-1}$ is also linear and continuous. Moreover there exists a constant $C>0$ such that

$$
\|[v]\|_{W^{2, p(\cdot)}(G) / W_{0}^{2, p(\cdot)}(G)} \leq C\left(\left\|g_{0}\right\|_{\operatorname{Tr}\left(W^{2, p(\cdot)}(G)\right)}+\left\|g_{1}\right\|_{\operatorname{Tr}\left(W^{1, p(\cdot)}(G)\right)}\right) .
$$

We show that $\|[v]\|_{W^{2, p(\cdot)}(G) / W_{0}^{2, p(\cdot)}(G)}=\inf \left\{\|v+W\|_{W^{2, p(\cdot)}(G)} ; w \in W_{0}^{2, p(\cdot)}(G)\right\}$ is achieved. Indeed, choose $w_{j} \in W_{0}^{2, p(\cdot)}(G)$ such that

$$
\lim _{j \rightarrow \infty}\left\|v+W_{j}\right\|_{W^{2, p(\cdot)}(G)}=\|[v]\|_{W^{2, p(\cdot)}(G) / W_{0}^{2, p(\cdot)}(G)} .
$$

Then $\left\{w_{j}\right\}$ is bounded in a reflexive Banach space $W_{0}^{2, p(\cdot)}(G)$. Passing to a subsequence, we may assume that $w_{j} \rightarrow w$ weakly in $W^{2, p(\cdot)}(G)$. Hence

$$
\|v+W\|_{W^{2, p(\cdot)}(G)} \leq \liminf _{j \rightarrow \infty}\left\|u+W_{j}\right\|_{W^{2, p(\cdot)}(G)}=\|[v]\|_{W^{2, p(\cdot)}(G) / W_{0}^{2, p(\cdot)}(G)} .
$$

If we put $u=v+w \in W^{2, p(\cdot)}(G)$, then we have $\gamma_{0}(u)=g_{0}, \gamma_{1}(u)=g_{1}$ and the estimate (7.13) holds.

The following Lemma is the celebrated Héron formula (cf. Amrouche and Girault [2, Lemma 3.5]).

Lemma 7.7. Let $G$ be a bounded domain of $\mathbb{R}^{d}$ with a $C^{1,1}$-boundary $\Gamma$ and let $p \in \mathcal{P}_{+}^{\log }(G)$. Then for $\boldsymbol{u} \in \boldsymbol{W}^{2, p(\cdot)}(G)$, the following Héron formula holds.

$$
\gamma_{0}(\operatorname{div} \boldsymbol{u})=\operatorname{div} \Gamma\left(\gamma_{0}(\boldsymbol{u})_{t}\right)+\gamma_{1}(\boldsymbol{u}) \cdot \boldsymbol{n}-2 K \gamma_{0}(\boldsymbol{u}) \cdot \boldsymbol{n},
$$

where $K$ denotes the mean curvature of $\Gamma$, $\operatorname{div}_{\Gamma}$ is the surface divergence and $\boldsymbol{v}_{t}=\boldsymbol{v}-(\boldsymbol{v} \cdot \boldsymbol{n}) \boldsymbol{n}$ is the tangent component of $\boldsymbol{v}$. 
Proposition 7.8. Let $G$ be a bounded domain of $\mathbb{R}^{d}$ with a $C^{1,1}$-boundary $\Gamma$ and let $p \in \mathcal{P}_{+}^{\log }(G)$. Then for every $\boldsymbol{g} \in \operatorname{Tr}\left(\boldsymbol{W}^{2, p(\cdot)}(G)\right)$ and $\varphi \in \operatorname{Tr}\left(W^{1, p(\cdot)}(G)\right)$, there exists $\boldsymbol{u} \in \boldsymbol{W}^{2, p(\cdot)}(G)$ such that $\gamma_{0}(\operatorname{div} \boldsymbol{u})=\varphi$ and $\gamma_{0}(\boldsymbol{u})=\boldsymbol{g}$, and there exists a constant $C>0$ depending only on $p$ and $G$ such that

$$
\|\boldsymbol{u}\|_{\boldsymbol{W}^{2, p(\cdot)}(G)} \leq C\left(\|\boldsymbol{g}\|_{\operatorname{Tr}\left(\boldsymbol{W}^{2, p(\cdot)}(G)\right)}+\|\varphi\|_{\operatorname{Tr}\left(W^{1, p(\cdot)}(G)\right)}\right) .
$$

Proof. Put $\boldsymbol{g}_{0}=\boldsymbol{g} \in \operatorname{Tr}\left(\boldsymbol{W}^{2, p(\cdot)}(G)\right), \boldsymbol{g}_{1}=2 K \boldsymbol{g}-\boldsymbol{n} \operatorname{div} \Gamma\left(\boldsymbol{g}_{t}\right)+\boldsymbol{\varphi} \boldsymbol{n}$. By Lemma 7.6, there exists $\boldsymbol{u} \in \boldsymbol{W}^{2, p(\cdot)}(G)$ such that $\gamma_{0}(\boldsymbol{u})=\boldsymbol{g}, \gamma_{1}(\boldsymbol{u})=2 K \boldsymbol{g}-\boldsymbol{n} \operatorname{div}_{\Gamma}\left(\boldsymbol{g}_{t}\right)+\varphi \boldsymbol{n}$, and (7.14) holds. Then by Lemma 7.7, we have $\gamma_{0}(\operatorname{div} \boldsymbol{u})=\varphi$.

Proposition 7.9. Let $G$ be a bounded domain of $\mathbb{R}^{d}$ with a $C^{1,1}$-boundary $\Gamma$ and let $p \in \mathcal{P}_{+}^{\log }(G)$. For any $\boldsymbol{g} \in \operatorname{Tr}\left(\boldsymbol{W}^{2, p(\cdot)}(G)\right)$ and any $\varphi \in W^{1, p(\cdot)}(G)$ satisfying the compatibility condition (7.10), there exists $\boldsymbol{u}_{0} \in \boldsymbol{W}^{2, p(\cdot)}(G)$ such that $\operatorname{div} \boldsymbol{u}_{0}=\varphi$ in $G$ and $\gamma_{0}\left(\boldsymbol{u}_{0}\right)=\boldsymbol{g}$, moreover, there exists a constant $C>0$ depending only on $p, d$ and $G$ such that

$$
\left\|\boldsymbol{u}_{0}\right\|_{W^{2, p(\cdot)}(G)} \leq C\left(\|\varphi\|_{W^{1, p(\cdot)}(G)}+\|\boldsymbol{g}\|_{\operatorname{Tr}\left(W^{2, p(\cdot)}(G)\right)}\right) .
$$

Proof. By Proposition 7.8, there exists $\boldsymbol{u} \in \boldsymbol{W}^{2, p(\cdot)}(G)$ such that $\gamma_{0}(\operatorname{div} \boldsymbol{u})=\gamma_{0}(\varphi), \gamma_{0}(\boldsymbol{u})=\boldsymbol{g}$ and (7.14) holds. Then $\operatorname{div} \boldsymbol{u}-\varphi \in W^{1, p(\cdot)}(G)$. Since $\gamma_{0}(\operatorname{div} \boldsymbol{u}-\varphi)=0$, we see that $\operatorname{div} \boldsymbol{u}-\varphi \in W_{0}^{1, p(\cdot)}(G)$. Since it follows from the compatibility condition (7.10) and the Green theorem that

$$
\int_{G}(\operatorname{div} \boldsymbol{u}-\varphi) d x=\int_{\Gamma} \boldsymbol{g} \cdot \boldsymbol{n} d \sigma-\int_{G} \varphi d x=0 .
$$

By [3, 4, Theorem 3.1] (e) (cf. Aramaki [6] for the case $p(\cdot)=p=$ const.), there exists $w \in$ $W_{0}^{2, p(\cdot)}(G)$, unique up to an additive function of $\operatorname{Kerdiv}:=\left\{\boldsymbol{v} \in \boldsymbol{W}_{0}^{2, p(\cdot)}(G)\right.$; $\operatorname{div} \boldsymbol{v}=0$ in $\left.G\right\}$, such that $\operatorname{div} \boldsymbol{w}=\operatorname{div} \boldsymbol{u}-\varphi$ in $G$, and there exists a constant $C>0$ such that

$$
\|[\boldsymbol{w}]\|_{\boldsymbol{W}^{2, p(\cdot)}(G) / \text { Kerdiv }} \leq C\|\operatorname{div} \boldsymbol{u}-\varphi\|_{W^{1, p(\cdot)}(G)} \leq C_{1}\left(\|\boldsymbol{g}\|_{\operatorname{Tr}\left(\boldsymbol{W}^{2, p(\cdot)}(G)\right)}+\|\varphi\|_{W^{1, p(\cdot)}(G)}\right) .
$$

Since we can easily see that

$$
\|[\boldsymbol{w}]\|_{\boldsymbol{W}^{2, p(\cdot)}(G) / \text { Kerdiv }}=\inf \left\{\|\boldsymbol{w}+\boldsymbol{v}\|_{\boldsymbol{W}_{0}^{2, p(\cdot)}(G)} \text { with } \operatorname{div} \boldsymbol{v}=0 \text { in } G\right\}
$$

is achieved, there exists $\boldsymbol{u}_{1} \in \boldsymbol{W}_{0}^{2, p(\cdot)}(G)$ such that

$$
\left\|\boldsymbol{w}+\boldsymbol{u}_{1}\right\|_{\boldsymbol{W}^{2, p(\cdot)}(G)}=\|[\boldsymbol{w}]\|_{\boldsymbol{W}^{2, p(\cdot)}(G) / \operatorname{Kerdiv}} \leq C_{1}\left(\|\boldsymbol{g}\|_{\operatorname{Tr}\left(\boldsymbol{W}^{2, p(\cdot)}(G)\right)}+\|\varphi\|_{W^{1, p(\cdot)}(G)}\right) .
$$

It suffices to put $\boldsymbol{u}_{0}=\boldsymbol{w}+\boldsymbol{u}_{1}$.

Proof of Theorem 7.4

By Proposition 7.9 , there exists $\boldsymbol{u}_{0} \in \boldsymbol{W}^{2, p(\cdot)}(G)$ such that

$$
\operatorname{div} \boldsymbol{u}_{0}=\varphi \text { in } G \text { and } \gamma_{0}\left(\boldsymbol{u}_{0}\right)=\boldsymbol{g}
$$


and the estimate $(7.15)$ holds. Moreover, there exists $\tilde{\pi} \in W^{1, p(\cdot)}(G)$ such that $\gamma_{0}(\widetilde{\pi})=\pi_{0}$ and

$$
\|\widetilde{\pi}\|_{W^{1, p(\cdot)}(G)} \leq C\left\|\pi_{0}\right\|_{\operatorname{Tr}\left(W^{1, p(\cdot)}(G)\right)} .
$$

If we put $\boldsymbol{v}=\boldsymbol{u}-\boldsymbol{u}_{0}$ and $\widehat{\pi}=\pi-\widetilde{\pi}$ in problem (7.9), then the system (7.9) is reduced to the following problem

$$
\begin{cases}-\Delta \boldsymbol{v}+\nabla \widehat{\pi}=\boldsymbol{f}+\Delta \boldsymbol{u}_{0}+\nabla \widetilde{\pi} & \text { in } G, \\ \operatorname{div} \boldsymbol{v}=0 & \text { in } G, \\ \boldsymbol{v}=0 & \text { on } \Gamma, \\ \widehat{\pi}=0 & \text { on } \Gamma .\end{cases}
$$

From Theorem 7.1, the estimates (7.15) and (7.16), the conclusion is clear.

Conflicts of Interest: The author(s) declare that there are no conflicts of interest regarding the publication of this paper.

\section{References}

[1] C. Amrouche, N.H. Seloula, $L^{p}$-theory for vector potentials and Sobolev's inequalities for vector fields: application to the Stokes equations with pressure boundary conditions, Math. Models Methods Appl. Sci. 23 (2013), 37-92. https://doi.org/10.1142/S0218202512500455.

[2] C. Amrouche, V. Girault, Decomposition of vector spaces and application to the Stokes problem in arbitrary dimension, Czech. Math. J. 44 (1994), 109-140.

[3] J. Aramaki, Equivalent relations with the J. L. Lions lemma in a variable exponent Sobolev space and an application to the Korn inequality, submitted.

[4] J. Aramaki, Equivalent relations with the J. L. Lions lemma in a variable exponent Sobolev space and their applications, submitted.

[5] J. Aramaki, An extension of a variational inequality in the Simader theorem to a variational exponent Sobolev space and applications: The Neumann case, submitted.

[6] J. Aramaki, On the J.L. Lions Lemma and its Applications to the Maxwell-Stokes Type Problem and the Korn Inequality, Commun. Math. Res. 37 (2021), 209-235. https://doi.org/10.4208/cmr. 2020-0043.

[7] F. Boyer and P. Fabrie, Mathematical Tools for the Study of the Incompressible Navier-Stokes Equations and Related Models, Springer, New York, 2010.

[8] P.G. Ciarlet, G. Dinca, A Poincaré inequality in a Sobolev space with a variable exponent, Chin. Ann. Math. Ser. B. 32 (2011), 333-342. https://doi.org/10.1007/s11401-011-0648-1.

[9] S.G. Deng, Eigenvalues of the $p(x)$-Laplacian Steklov problem, J. Math. Anal. Appl. 339 (2008), 925-937. https: //doi.org/10.1016/j.jmaa.2007.07.028.

[10] L. Diening, Theoretical and numerical results for electrorheological fluids, PH.D. Thesis, University of Freiburg, Germany 2002.

[11] L. Diening, P. Harjulehto, P. Hästö and M. Rǔžička, Lebesgue and Sobolev Spaces with Variable Exponent. Lecture Notes in Math., Springer, Berlin, 2017.

[12] X.-L. Fan, Q.-H. Zhang, Existence of solutions for $p(x)$-Laplacian Dirichlet problem, Nonlinear Anal.: Theory Methods Appl. 52 (2003), 1843-1852. https://doi.org/10.1016/S0362-546X (02)00150-5.

[13] X. Fan, Q. Zhang, D. Zhao, Eigenvalues of $p(x)$-Laplacian Dirichlet problem, J. Math. Anal. Appl. 302 (2005), 306-317. https://doi.org/10.1016/j.jmaa.2003.11.020. 
[14] X. Fan, D. Zhao, On the spaces $L^{p(x)}(\Omega)$ and $W^{m, p(x)}(\Omega)$, J. Math. Anal. Appl. 263 (2001), 424-446. https: //doi.org/10.1006/jmaa.2000.7617.

[15] D. Fujiwara, H. Morimoto, An $L_{r}$-theorem of the Helmholtz decomposition of vector fields, J. Fac. Sci. Univ. Tokyo, Sec. I, 24 (1977), 685-700.

[16] G. Galdi. An introduction to the mathematical theory of the Navier-Stokes equations, Linearized Steady Problem, Vol. 38 of Tracts in Natural Philosophy, Springer, New York, 1994.

[17] D. Gilbarg, N.S. Trudinger, Elliptic partial differential equations of second order, Springer, Berlin, Heidelberg, New York, 1998.

[18] T.C. Halsey, Electrorheological fluids, Science. 258 (1992), 761-766. https://doi.org/10.1126/science.258. 5083.761.

[19] O. Kováčik, J. Rákosnic, On spaces $L^{p(x)}(\Omega)$ and $W^{k, p(x)}(\Omega)$, Czechoslovak Math. J. 41 (1991), $592-618$.

[20] H. Kozono, T. Yanagisawa, Global div-curl lemma on bounded domains in $\mathbb{R}^{3}$, J. Funct. Anal. 256 (2009), $3847-$ 3859.

[21] M. Mihăilescu, V. Rădulescu, A multiplicity result for a nonlinear degenerate problem arising in the theory of electrorheological fluids, Proc. R. Soc. A. 462 (2006), 2625-2641. https://doi .org/10.1098/rspa.2005.1633.

[22] T. Miyakawa, On nonstationary solutions of the Navier-Stokes equations in an exterior domain, Hiroshima Math. J. 12 (1982), 115-140.

[23] M. Růžička, Electrotheological fluids: Modeling and mathematical theory, Lecture Notes in Mathematics, Vol. 1784, Berlin, Springer-Verlag, 2000.

[24] C.G. Simader, H. Sohr, A new approach to the Helmholtz decomposition and the Neumann problem in $L^{q}$-spaces for bounded and exterior domains, in: G.P. Galdi (Ed.), Mathematical Problem Relating to the Navier-Stokes Equations, in; Ser. Adv. Math. Appl. Sci., World Scientific, Singapore, New Jersey, London, Hong Kong, (1992), $1-35$.

[25] C.G. Simader, The Dirichlet problem for the laplacian in bounded and unbounded domains, Pitman Res. Noes Math. Ser., Vol. 360, Longman, 1996,

[26] Z. Wei, Z. Chen, Existence results for the $p(x)$-Laplacian with nonlinear boundary condition, ISRN Appl. Math. 2012 (2012), 727398.

[27] Z. Yücedağ, Solutions of nonlinear problems involving $p(x)$-Laplacian operator, Adv. Nonlinear Anal. 4 (2015), 285-293. https://doi.org/10.1515/anona-2015-0044.

[28] D. Zhao, W.J. Qiang, X.L. Fan, On generalized Orlicz space $L^{p(x)}(\Omega)$, J. Gansu Sci. 9 (1996), 1-7 (in Chinese).

[29] V.V. Zhikov, Averaging of functionals of the calculus of variations and elasticity theory, Math. USSR Izv. 29 (1987), 33-66. https : //doi.org/10.1070/IM1987v029n01ABEH000958. 